\title{
Abnormal Pressure Dependence of the Phase Boundaries in PEE-PDMS and PEP-PDMS Binary Homopolymer Blends and Diblock Copolymers
}

\section{Dietmar Schwahn, ${ }^{,+\dagger}$ Henrich Frielinghaus, ${ }^{\dagger, \neq}$ Kell Mortensen, ${ }^{\ddagger}$ and Kristoffer Almdal ${ }^{\ddagger}$}

Forschungszentrum J ülich $\mathrm{GmbH}$, Institut für Festkörperforschung, D-52425 J ülich, Germany, and Risø National Laboratory, DK-4000 Roskilde, Denmark

Received February 10, 2000; Revised Manuscript Received May 23, 2000

\begin{abstract}
Both polymer blends and diblock copolymers have been investigated with small-angle neutron scattering (SANS) in varying temperature and pressure fields. Four samples were studied: a polymer blend of near critical composition and the corresponding symmetric diblock copolymer of poly(ethylethylene) (PEE) and poly(dimethyl siloxane) (PDMS) and two further symmetric diblock copol ymers of poly(ethylenepropylene) (PEP) and PDMS differing in their molecular mass. From the SANS results the phase transition temperatures, the Flory-Huggins interaction parameter, the Ginzburg number, and the sizes of the chain were determined. As the transition temperature and the Flory-Huggins parameter are independently determined from the SANS data, the Clausius-Clapeyron equation offers a cross-check of the theoretical background used for SANS analysis. The resulting parameters showed for all samples qualitatively similar behavior. In particular, a quite unusual decrease of the phase boundaries in low increasing pressure regimes was observed. Analysis based on the Clausius-Clapeyron equation shows that the reason for this pressure-induced decrease of the phase boundary is a dominating increase of the entropic Flory-Huggins parameter. The Ginzburg parameter was found constant with pressure. The size of the diblock copolymer chains changes with temperature and pressure. Beyond the chain stretching observed near the ordering temperatures ( $\mathrm{T}_{\mathrm{ODT}}$ ) for both decreasing temperature and applied pressure, a discontinuous decrease of the chain size was consistently found at the TODT. The chain size vs temperature was found to follow a scaling behavior with slightly different exponents in the disordered and the ordered regimes consistent with former simulation calculations.
\end{abstract}

\section{Introduction}

In this publication we present small-angle neutron scattering (SANS) experiments on a binary polymer blend of near critical composition and on three symmetric diblock copolymers; we have studied and compared their behavior in varying external pressure and temperature fields. The thermodynamic behavior of polymer blends is well understood in terms of the mean field Flory-Huggins theory and their deviations near the critical point when thermal composition fluctuations become dominant. ${ }^{1-3}$ This is not yet the case for diblock copolymers; thermal composition fluctuations play a much more dominant role as diblock copol ymers bel ong to the Brazovskiî universality class. ${ }^{4-10} \mathrm{~A}$ mean field theory within the random phase approximation was derived by Leibler ${ }^{7}$ and Ohta and Kawasaki ${ }^{8}$ and was later on extended by F redrickson and Helfand ${ }^{9,10}$ taking thermal fluctuations into consideration. The last mentioned theoretical approach is valid in the weak segregation regime if the degree of thermal fluctuations is not too large and which should be fulfilled for molecular chains larger than degree of polymerization $\mathrm{N}=10^{4}$, at least for asymmetric polymers. ${ }^{9}$ The polymers studied by us, however, are characterized by $\mathrm{N}$ values of only 80-170 segments. Nevertheless, we apply this theory for the interpretation of our experimental results, as it is presently the most devel oped one for diblock copolymers.

† Institut für F estkörperforschung.

₹ Risø National Laboratory.

* To whom correspondence should be addressed.
An important part of this publication is related to investigations of sample properties in an external pressure field. Most studies of polymer blends so far show an increase of the phase boundary with pressure which is quite obvious as the free volume decreases with pressure and thereby diminish the entropy of mixing. ${ }^{11-15}$ Such a behavior was also predicted theoretically for both polymer blends and diblock copolymers, as based on lattice cluster theory (LCT) calculation made by Dudowicz and Freed. ${ }^{16}$ The situation, however, was for a long time not so clear for diblock copolymers; an abnormal decrease of the disorder-order phase boundary in low-pressure fields was reported for the first studied samples. ${ }^{17-21}$ The results of the PS-PI diblock in ref 17 were supplemented later on by experiments in lower pressure fields. ${ }^{19,20}$ In ref 19 the abnormal behavior was indirectly concluded from the ClausiusClapeyron equation after measuring the density discontinuity at the phase transition and the enthal py of mixing; this prediction was later confirmed in a scattering experiment. ${ }^{20}$ In the meantime such an abnormal behavior of the phase boundary was also found for a sample of PEMS/PDMS blend,22 and experiments on samples of corresponding PB/PS blend and diblock copolymers both show a normal pressure behavior with respect to pressure. ${ }^{15}$

The studies presented here were performed on a binary polymer blend and three diblock copolymers with three different monomers. The phase boundaries, the Flory-Huggins $(\mathrm{FH})$ interaction parameters, and the Ginzburg numbers are determined. Further, the chain compressibilities are evaluated from the experimental 
results. With the application of the Clausius-Clapeyron equation we have furthermore compared the pressuredependent phase boundary with those evaluated from the FH parameter. This allows an independent crosscheck of the $\mathrm{FH}$ parameter as determined from the theoretical approaches of Flory-Huggins and Fredrickson-Helfand for blends and diblock copolymers, respectively. Finally, we will discuss the temperature dependence of the chain conformation and compressibility. Preliminary parts of this work have already been published in ref 21.

\section{Theoretical Background}

In this section we give a short review of the theoretical background which is needed for the interpretations of our experiments. The relevant thermodynamic potential here is the Gibbs free energy of mixing $G_{m}(T, P, \Phi)$ because the polymer melt samples (one binary homopolymer blend and three diblock copolymers) were studied at fixed composition $\Phi$ in the external fields of temperature $T$ and pressure $P$. We will discuss the blend only in the framework of the mean field Flory-Huggins $(\mathrm{FH})$ model because the temperature steps near the phase boundary were not sufficiently narrow to resolve the fluctuation effects. The FH theory represents a mean field approximation neglecting the effects of thermal fluctuations. The theoretical approach for diblock copolymers is still insufficient as can be understood from the following arguments. While thermal fluctuation effects in polymer blends are described within the universality class of the 3D I sing model, ${ }^{1-3}$ diblock copolymers bel ong to the Brazovskiî universality class. ${ }^{2,4-10} \mathrm{~A}$ characteristic behavior of the Brazovskiî universality class is the strong influence of thermal composition fluctuations which even leads to a weak first-order phase transition. ${ }^{6}$ We interpret our data with the Fredrickson-Helfand model; this is the so far best devel oped theory for large polymer chains $\left(\mathrm{N} \geq 10^{4}\right.$; for symmetric diblock copolymers the theory seems to be valid even at lower $\mathrm{N}$ ) in the so-called weak segregation regime. ${ }^{9,10}$ Even though our data are rather well described by this theory, one has to be aware that the size of our diblock copolymer systems, $\mathrm{N} \cong 80-170$, are 2 orders of magnitude smaller than $\mathrm{N} \cong 10^{4}$.

\section{A. The Clausius-Clapeyron Equation and the} Flory-Huggins Model. The Clausius-Clapeyron equation describes the coexistence line of two phases in a $\mathrm{T}-\mathrm{P}$ plane according to

$$
\Delta \mathrm{T}_{\mathrm{B}} / \Delta \mathrm{P}=\mathrm{T}_{\mathrm{B}} \mathrm{V}_{\mathrm{m}} / \mathrm{H}_{\mathrm{m}}
$$

e.g., the change of the phase transition temperature $T_{B}$ with pressure is related to the volume $V_{m}$ and enthal py $\mathrm{H}_{\mathrm{m}}$ differences of the two phases. ${ }^{24} \mathrm{~F}$ or further analysis the Gibbs free energy of mixing is needed

$$
G_{m}(T, P, \Phi)=G_{12}-\Phi G_{1}+(1-\Phi) G_{2}=H_{m}-T S_{m}
$$

with the symbols $\Phi$ representing the volume fraction of the component " 1 ", $G_{1}$ and $G_{2}$ the Gibbs potentials of the two components, and $\mathrm{G}_{1,2}$ that of the mixture. Within the mean field theory of polymer blends described by the Flory-Huggins ( $\mathrm{FH}$ ) model the enthalpy and entropy of mixing are given as

$$
\mathrm{H}_{\mathrm{m}} / \mathrm{RT}=\Phi(1-\Phi) \chi_{\mathrm{h}} / T
$$

and

$$
\begin{array}{r}
\mathrm{S}_{\mathrm{m}}=-\left\{\left(\Phi N_{1}\right) \ln \Phi+[(1-\Phi)] \mathrm{N}_{2} \ln (1-\Phi)\right\}+ \\
\Phi(1-\Phi) \chi_{\sigma}
\end{array}
$$

with the molar vol umes $\mathrm{V}_{1}$ and $\mathrm{V}_{2}$ of the two molecular species. ${ }^{1,2}$ The first term of $\Delta S_{m}$ is the configurational part while the second one contains all the other entropic contributions related to polymer end effects, compressibility, and other ones. The entropic term $\chi_{\sigma}$ is a phenomenological parameter, which had to be included in order to describe experimental observations as obtained mainly from scattering experiments. Usually, the enthal pic and entropic terms $\chi_{\mathrm{h}}$ and $\chi_{\sigma}$ are comprised within the $\mathrm{FH}$ interaction parameter according to $\chi=$ $\chi_{\mathrm{h}} / \mathrm{T}-\chi_{\sigma}$. As a mean field theory the $\mathrm{FH}$ model is valid only far from the critical temperature of phase decomposition where the thermal compositi on fluctuations are sufficiently weak to be described within the Gaussian approximation; as determined by the Ginzburg criterion, the mean field approximation becomes invalid near the critical temperature when the thermal fluctuations become strong and correlated. ${ }^{1-3,25,26}$

The Gibbs free energy of mixing $G_{m}(T, P, \Phi)$ contains all thermodynamic information. The susceptibility obtained in a scattering experiment is related to its second derivative with respect to composition. This also means that in scattering experiments one measures an effective FH parameter $\Gamma$ defined as $\Gamma \equiv \partial^{2}[\phi(1-\phi) \chi] / \partial \phi^{2}$. The effective $\mathrm{FH}$ parameter $\Gamma$ is given in units $\left[\mathrm{mol} / \mathrm{cm}^{3}\right]$, and the product of $(\Gamma \mathrm{V})$ has the same meaning as the product $(\chi N)$, which is frequently discussed in corresponding SANS and theoretical papers. From now on we will denote $\Gamma$ the $\mathrm{FH}$ parameter, since this is the parameter that is obtained in the scattering experiments. In the case of a symmetrical systems with volume $\mathrm{V}=\mathrm{V}_{1}=\mathrm{V}_{2}$ the critical composition is equal to $\Phi_{\mathrm{c}}=0.5$. F or a homopolymer blend, the FH parameter is $\Gamma_{c}=2 N$ at the critical temperature, $T_{c}$, while for a symmetric diblock copolymer, as derived below, it is $\Gamma_{c}$ $\cong 10.495 \mathrm{~N}$.

The Clausius-Clapeyron equation for polymer blends and diblock copolymers can now be written as ${ }^{15}$

$$
\Delta \mathrm{T}_{\mathrm{B}} / \Delta \mathrm{P}=\mathrm{T}_{\mathrm{B}}\left(\partial \Gamma_{\mathrm{h}} / \partial \mathrm{P}-\mathrm{T}_{\mathrm{B}} \partial \Gamma_{\sigma} / \partial \mathrm{P}\right) / \Gamma_{\mathrm{h}}-\mathrm{K} \partial \mathrm{Gi} / \partial \mathrm{P}
$$

The first term has been derived from $H_{m}, V_{m}=\partial G_{m} / \partial P$, and their definitions in eqs $2-4$ assuming a constant $\Gamma$ with respect to composition (i.e., $\Gamma \equiv \chi \mathrm{N} / \mathrm{N}$ ). The second term describes the stabilization effect of thermal fluctuations described by the Ginzburg number Gi. This term, however, will not be relevant here as the experiments will show a pressure-independent $\mathrm{Gi}$ for all samples.

B. Structure Factor of a Noninteracting Polymer Blend and Diblock Copolymer. The structure factor $\mathrm{S}(\mathrm{Q})$ of two linear noninteracting homopolymers of the same molar volume $\mathrm{V}$ and mixed with a volume fraction $\Phi$ is given as

$$
\mathrm{S}(\mathrm{Q})=\Phi(1-\Phi) \operatorname{VP}\left(\mathrm{QR}_{\mathrm{g}}\right)
$$

For chains obeying Gaussian statistics the intramolecular form factor $\mathrm{P}(\mathrm{Q})$ is given within the Debye approximation as $\mathrm{P}_{\mathrm{DB}}(\mathrm{x})=2[\mathrm{x}-1+\exp (-\mathrm{x})] / \mathrm{x}^{2}$ with $\mathrm{x}=$ $\mathrm{R}_{\mathrm{g}}^{2} \mathrm{Q}^{2}\left(\mathrm{R}_{\mathrm{g}}\right.$ radius of gyration). ${ }^{27}$ The maximum of $\mathrm{S}(\mathrm{Q})$ for homopolymer mixture is observed at $Q=0$, giving 
$\mathrm{S}(0)=\Phi(1-\Phi) \mathrm{V}$. In diblock copolymers, on the other hand, $S(Q)$ shows a maximum at a finite wavenumber $\mathrm{Q}=\mathrm{Q}_{\mathrm{m}}$ reflecting the constraint that composition fluctuations can only occur on the length scale of the polymer chain.

The structure factor of symmetric $(f=0.5)$ diblock copolymers is determined by the difference of $P(Q)$ of one block $\left(\mathrm{P}_{\mathrm{B}}\right)$ and of the whole chain $\left(\mathrm{P}_{\mathrm{T}}\right)$ according to ${ }^{27}$

$$
S(Q)=(V / 4)\left[P_{B}(Q)-P_{T}(Q)\right]
$$

At $\mathrm{Q}=0$ both $\mathrm{P}(\mathrm{Q}=0)=1$ cancel each other, giving $\mathrm{S}(0)$ $=0$ while the interference peak occurs at $\mathrm{Q}_{\mathrm{m}} \cong 1.945 /$ $\mathrm{R}_{\mathrm{g}}$ and $\mathrm{S}\left(\mathrm{Q}_{\mathrm{m}}\right) \cong 0.1906(\mathrm{~V} / 4)$ because of the stronger decrease of $\mathrm{P}_{\mathrm{T}}$ with $\mathrm{Q}$. In the following we will make use of an approximation of $\mathrm{S}(\mathrm{Q})$ obtained from an expansion around $\mathrm{Q}_{m}$ according to ${ }^{8}$

$$
\mathrm{S}(\mathrm{Q}) \cong \mathrm{V}\left[2\left(\Gamma_{\mathrm{S}} \mathrm{V}\right)+\mathrm{e}\left(\mathrm{Q}^{2}-\mathrm{Q}_{\mathrm{m}}^{2}\right)^{2}\right]^{-1}
$$

with $\Gamma_{\mathrm{S}} \mathrm{V}=10.495$ and $\mathrm{e} \cong 0.482 \mathrm{R}_{\mathrm{g}}^{4}$. $\Gamma_{\mathrm{S}}$ is the FloryHuggins parameter at the spinodal or critical temperature as will be discussed below.

C. Structure Factor of Diblock Copolymers. Usually a repulsive interaction energy is existing between the two blocks leading to thermal fluctuations and transitions of ordered phases at low temperatures. An effective Hamiltonian has been expressed for symmetric ( $f=0.5$ ) diblock copolymers within the Hartree approximation and neglecting thermal composition fluctuations as $2,5,8,28,29$

$$
\begin{aligned}
\mathrm{H}\{\bar{\varphi}(\mathrm{x})\}=\int \mathrm{d}^{\mathrm{d}} \mathrm{x}\left\{\frac{1}{2} \bar{\varphi}\left[\mathrm{r}_{\mathrm{R}}+\mathrm{e}\left(\nabla^{2}+\mathrm{Q}_{\mathrm{m}}^{2}\right)^{2}\right] \bar{\varphi}+\right. \\
\left.\left(\mathrm{u}_{\mathrm{R}} / 4 !\right) \bar{\varphi}^{4}+\left(\mathrm{w}_{\mathrm{R}} / 6 !\right) \bar{\varphi}^{6}\right\}
\end{aligned}
$$

with the order parameter $\bar{\varphi}(\mathrm{x})$, which becomes zero in the disordered phase. The effects of thermal fluctuations can be included in the parameters evaluated from a one loop renormalization procedure. The parameter of the first term in eq 9 is, when transformed into the Q-space representation, the inverse structure factor as given in eq 8, but with a renormalized $\mathrm{FH}$ parameter $\Gamma_{\mathrm{R}}$ in $\mathrm{S}\left(\mathrm{Q}_{\mathrm{m}}\right)$ according to

$$
\mathrm{S}^{-1}\left(\mathrm{Q}_{\mathrm{m}}\right) \equiv \mathrm{r}_{\mathrm{R}}=2\left[\Gamma_{\mathrm{S}} \mathrm{V}-\Gamma_{\mathrm{R}} \mathrm{V}\right] \mathrm{V}
$$

The structure factor at $Q=Q_{m}$ represents a susceptibility that becomes singular at $\Gamma_{\mathrm{R}}=\Gamma_{\mathrm{s}}$. In diblock systems the influence of thermal fluctuations is stronger than in blends because integrals in the renormalization procedure become singular within a shell of the $\mathrm{Q}$ space at $\mathrm{Q}_{\mathrm{m}}{ }^{2,6,10}$ The renormalized $\Gamma_{\mathrm{R}}$ has been derived by Fredrickson-Helfand, ${ }^{9,10}$ including the effect of thermal fluctuations according to

$$
\Gamma_{\mathrm{R}} \mathrm{V}=\Gamma \mathrm{V}-\mathrm{Gi} \sqrt{\mathrm{S}\left(\mathrm{Q}_{\mathrm{m}}\right) / \mathrm{V}}
$$

The "fluctuation" term is proportional to a Ginzburg number $\mathrm{Gi}=\tilde{c} / \overline{\mathrm{N}}^{1 / 2}$ and to the square root of the susceptibility. The parameter $\tilde{c}$ depends on molecular parameters and is $\tilde{c}=105.7$ for a chain length ratio $f=$

\begin{tabular}{|c|c|c|c|c|}
\hline sample & $\begin{array}{l}\text { PEP- } \\
\text { PDMS (1) }\end{array}$ & $\begin{array}{c}\text { PEP- } \\
\text { PDMS (2) }\end{array}$ & $\begin{array}{l}\text { PEE- } \\
\text { PDMS }\end{array}$ & $\begin{array}{l}\text { PEE/ } \\
\text { PDMS }\end{array}$ \\
\hline $\mathrm{X}^{*} \equiv\left(\mathrm{Q}^{*} \mathrm{R}_{\mathrm{g}}\right)^{2}$ & & 3.783 & & - \\
\hline $\mathrm{R}_{\mathrm{g}}=\sqrt{ } \mathrm{x}^{*} / \mathrm{Q}^{*}[\AA]$ & $\begin{array}{l}31.4 \\
\left(T=182^{\circ} \mathrm{C}\right)\end{array}$ & $\begin{array}{l}40.7 \\
\left(T=178^{\circ} \mathrm{C}\right)\end{array}$ & 48.9 & - \\
\hline $\begin{array}{l}\bar{N}=\left(R_{0}^{3} N\right)^{2} \\
\tilde{C}\end{array}$ & $5.13 \times 10^{3}$ & $\begin{array}{c}13.6 \times 10^{3} \\
105.7\end{array}$ & $20.5 \times 10^{3}$ & - \\
\hline Gi & 1.48 & 0.9 & 0.74 & - \\
\hline$\Gamma_{\mathrm{s}} \mathrm{V}$ & & 10.495 & & 2 \\
\hline ГODTV & 13.8 & 12.9 & 12.6 & - \\
\hline
\end{tabular}
0.5. ${ }^{9}$ The parameter $\bar{N}=\left(R_{0}^{3} N\right)^{2}\left(R_{0}^{2}=6 R_{g}^{2}\right.$ being the end-to-end distance of the polymer) is the average number of chains in the volume $\mathrm{R}_{0}^{3}{ }^{3}{ }^{10}$ The Ginzburg criterion foll ows the scal ing law according to $\mathrm{Gi} \propto \mathrm{V}^{-0.5}$,
Table 1. Evaluated Thermodynamic Parameters

indicating that thermal fluctuations have less influence in higher polymer weight systems. The theoretical parameters determining $\mathrm{Gi}$ have been collected for all diblock copolymer samples in Table 1.

In the limit of a negligible $\mathrm{Gi}$, the fluctuation effect becomes negligible and $\Gamma_{\mathrm{R}} \mathrm{V}=\Gamma \mathrm{V}$ becomes valid in accordance with the mean field approximation derived by Leibler. ${ }^{7}$ So, if fluctuations become relevant, the mean field or bare $\mathrm{FH}$ parameter $\Gamma$ in eq 11 is reduced by the fluctuation term and thereby stabilizes the disordered phase. The Hamiltonian given in eq 9 introduces the parameter $\Gamma$ as an enthalpic term according to $\Gamma_{\mathrm{h}} / T$. Experiments, however, have shown that similarly to polymer blends $\Gamma$ has to be extended by an entropic term $\Gamma_{\sigma}$ in order to describe the experimental data. So the $\mathrm{FH}$ parameter is a free energy parameter according to $\Gamma=\Gamma_{h} / T-\Gamma_{\sigma}{ }^{4}$

In symmetric diblock copolymers the transition from the disordered to the ordered phase is predicted as a second-order phase transition if one neglects the effect of thermal fluctuations. In the case of strong thermal fluctuations, however, the second term of the Hamiltonian in eq 9 becomes negative, indicating a transition of a weak first-order phase transition at the $\mathrm{FH}$ parameter. ${ }^{9}$

$$
\Gamma_{\mathrm{ODT}} \mathrm{V}=10.495+41(\mathrm{Gi} / \tilde{\mathrm{C}})^{2 / 3}
$$

At $\mathrm{Gi}=0$ a second-order phase transition would occur at $\Gamma_{\mathrm{ODT}} \mathrm{V} \equiv \Gamma_{\mathrm{S}} \mathrm{V}=10.495$; the second term in eq 12 describes the stabilization effect of the thermal fluctuations (see corresponding parameters in Table 1).

D. Structure Factor of Polymer Blends within the Flory-Huggins Approximation. The structure factor S(Q) of thermal composition fluctuations in binary polymer blends can be derived within the random phase approximation.1,2 It is a mean field approximation, which in the limit of small wavenumber $\mathrm{Q}$ is given as

$$
\mathrm{S}^{-1}(\mathrm{Q})=\mathrm{S}^{-1}(0)+\mathrm{AQ}^{2}
$$

For our purpose $\mathrm{S}(\mathrm{Q})$ in eq 13 describes the thermal composition fluctuations well as we always could perform our measurements at sufficiently small Q. So we obtain two parameters from the scattering experiments; the structure factor extrapolated to $Q=0$ is a susceptibility which, according to the fluctuation-dissipation theorem, is related to the Gibbs free energy of mixing, $\mathrm{G}_{\mathrm{m}}$ (eq 2$)$, according to $\mathrm{S}(0)=\partial^{2}\left(\mathrm{G}_{\mathrm{m}} / \mathrm{RT}\right) / \partial \Phi^{2}$ (R is the gas constant). ${ }^{1,2}$ Within the Flory-Huggins approximation (eqs 3 and 4 ) the susceptibility is obtained as

$$
\mathrm{S}^{-1}(0)=2\left[\Gamma_{\mathrm{C}} \mathrm{V}-\Gamma \mathrm{V}\right] / \mathrm{N}
$$

The FH parameter $\Gamma=\Gamma_{\mathrm{h}} / \mathrm{T}-\Gamma_{\sigma}$ has the same meaning as for diblock copolymers. The symbol $\Gamma_{\mathrm{C}}$ is the $\mathrm{FH}$ 
Table 2. Sample Characteristics of PEE-PDMS

\begin{tabular}{|c|c|c|c|c|}
\hline \multirow[b]{2}{*}{ polymer } & \multicolumn{2}{|c|}{ polymer blend } & \multicolumn{2}{|c|}{ diblock copolyme } \\
\hline & poly(ethylethylene) & poly(dimethylsiloxane) & poly(ethylethylene) & poly(dimethylsiloxane) \\
\hline chem structure & $\begin{array}{l}\mathrm{PEE} \\
\left(\mathrm{C}_{4} \mathrm{H}_{5.8} \mathrm{D}_{2.2}\right)\end{array}$ & $\begin{array}{l}\text { PDMS } \\
\left(\mathrm{SiOC}_{2} \mathrm{H}_{6}\right)\end{array}$ & $\begin{array}{l}\mathrm{PEE} \\
\left(\mathrm{C}_{4} \mathrm{H}_{5.8} \mathrm{D}_{2.2}\right)\end{array}$ & PDMS $\left(\mathrm{SiOC}_{2} \mathrm{H}_{6}\right)$ \\
\hline$\sigma[\AA]$ & 5.78 & 5.84 & 5.78 & 5.84 \\
\hline$\Omega\left[\mathrm{cm}^{3} / \mathrm{mol}\right]$ & 66 & 77.7 & 66 & 77.7 \\
\hline$\sum \mathrm{c}_{\mathrm{i}} \mathrm{b}_{\mathrm{i}} / \Omega_{\mathrm{i}}\left[10^{10} \mathrm{~cm}^{-2}\right]$ & 1.79 & 0.0628 & 1.79 & 0.0628 \\
\hline$\overline{\mathrm{V}}_{\mathrm{w}}\left[\mathrm{cm}^{3} / \mathrm{mol}\right]$ & 2010 & 2270 & \multirow{3}{*}{\multicolumn{2}{|c|}{$\begin{array}{c}12000 \\
168 \\
\mathrm{f}=0.5 \\
8.82\end{array}$}} \\
\hline & 30.5 & 29.2 & & \\
\hline $\begin{array}{l}\text { volume fraction PEE } \\
\Gamma_{\mathrm{s}}\left[10^{-4} \mathrm{~mol} / \mathrm{cm}^{3}\right]\end{array}$ & \multicolumn{2}{|c|}{$\begin{array}{c}\Phi=0.516 \\
9.37\end{array}$} & & \\
\hline
\end{tabular}

parameter at the critical point; for a symmetric mixture with $\mathrm{V}=\mathrm{V}_{\mathrm{A}}=\mathrm{V}_{\mathrm{B}}$ and the critical composition $\Phi_{\mathrm{C}}=$ 0.5 , one gets from the configurational entropy $\Gamma_{C}=2 \mathrm{~N}$. $\mathrm{So}$ in the case of a zero $\mathrm{FH}$ parameter S(0) in eq 14 is given as $\mathrm{S}(0)=\mathrm{V}\left[2\left(\Gamma_{\mathrm{s}} \mathrm{V}\right)\right]^{-1}$ and is identical to the value of $S\left(Q_{m}\right)$ in eq 10 for diblock copolymers. Both susceptibilities $S(0)$ and $S\left(Q_{m}\right)$ for $\Gamma=0$ are identical to those given in eqs 6 and 8, respectively, and from which $\Gamma_{C}$ can be evaluated. The second parameter $A$ in eq 3 is related to the correlation length of the thermal fluctuations according to $\xi=\sqrt{\mathrm{AS}(0)}$.

E. Thermodynamic Parameters Obtained from SANS. The experimental $\mathrm{FH}$ parameters $\Gamma=\Gamma^{\mathrm{H}}$ in eq 14 and $\Gamma=\Gamma^{\mathrm{DB}}$ in eq 11 are bare or mean field parameters of the Flory-Huggins theory valid at high temperatures when the reduced temperature, $t:=(T-$ $\left.T_{C}\right) / T$, is much larger than the Ginzburg number, $t \gg$ Gi. ${ }^{25}$ One implicit assumption of the theories discussed above is the equivalence of $\Gamma=\Gamma^{\mathrm{H}}=\Gamma^{\mathrm{DB}}$. In more sophisticated lattice cluster theory (LCT) calculations by Dudowicz and F reed, the following relationship was proposed

$$
\Gamma^{\mathrm{DB}}=\left(\Gamma_{\mathrm{h}}^{\mathrm{H}}-\mathrm{A}_{\mathrm{h}} \mathrm{N}\right) / \mathrm{T}-\left(\Gamma_{\sigma}^{\mathrm{H}}-\mathrm{A}_{\sigma} N\right)
$$

which contains an additional term associated with the junction region of diblock copolymers. ${ }^{30} \mathrm{~B}$ oth $\mathrm{V}^{-1}$ terms are of the order of magnitude larger than similar postulated terms related to the end groups of chains. ${ }^{30}$ So, according to eq 15 , the same $\mathrm{FH}$ parameter is expected only in the limit of large polymer chains in blends and diblock copolymers.

F. Characteristic Peak Position $\mathbf{Q}_{\mathbf{m}}$ of Diblock Copolymers. Within the classical theories for diblock copolymers by Leibler, ${ }^{7}$ Ohta-Kawasaki, ${ }^{8}$ and Fredrickson-Helfand ${ }^{9,10} \mathrm{Q}_{\mathrm{m}}$ was obtained as a constant value because of the assumptions of random phase approximation assuming a Gaussian coil conformation and of a local approximation of the higher order vertex functions $u_{R}$ and $w_{R}$ in the Hamiltonian of eq 9, taking these parameters as constants. Experiments, however, have shown that deviations from a Gaussian chain conformation al ready occurs well above the ODT transition. ${ }^{31}$ Theoretical extensions by Barrat and Fredrickson $^{32}$ and by Stepanow ${ }^{33}$ qualitatively confirm the experimental results. The theory by Stepanow ${ }^{33}$ going beyond the RPA approximation additionally proposes a stepwise increase of the coil size at the ODT transition in consistence with experiments by Stühn et al.; 34 on the other hand, in asymmetrical diblock copolymers a stepwise decrease of the coil was found by Ogawa et al. ${ }^{35}$

More quantitative results have been obtained from recent simulation studies by Fried and Binder;36 a scaling behavior of $\mathrm{Q}_{\mathrm{m}}$ vs $(\Gamma \mathrm{N})$ or $1 / \mathrm{T}$ at constant $\mathrm{N}$ was found with a maximum effective exponent $\Delta v \cong-0.24$ near the ODT transition instead of the predicted $\Delta v=$ 0 by the RPA. We furthermore define for our experimental analysis a chain compressibility $\beta_{\text {conf }}$ according to

$$
\beta_{\text {conf }}=-3 \partial \ln \mathrm{R}_{\mathrm{g}} / \partial \mathrm{P}=3 \partial \ln \mathrm{Q}_{\mathrm{m}} / \partial \mathrm{P}
$$

describing the pressure dependence of the measured position $\mathrm{Q}_{\mathrm{m}}$.

\section{Experimental Section}

The scattering experiments were performed at the SANS diffractometers at the DR3-reactor of the Risø National Laboratory ${ }^{37}$ and at the FRJ 2-reactor of the "F orschungszentrum J ülich". ${ }^{38}$ F or these studies a steel-bodi ed temperaturepressure cell was used which allows an in-situ change of pressure and temperature in the range of $0.1 \leq \mathrm{P}(\mathrm{MPa}) \leq$ 200 and $-20 \leq \mathrm{T}\left({ }^{\circ} \mathrm{C}\right) \leq 200$, respectively, with a temperature control better than $0.01 \mathrm{~K}$. This cell was developed in our laboratory in J ülich. The sample thickness and its neutronirradiated diameter was $0.1 \mathrm{~cm}$ and $0.7 \mathrm{~cm}$, respectively. After each change of the temperature we waited for half an hour before proceeding with the neutron measurements in order to safely achieve a stationary temperature field in the relatively large pressure cell. The temperature of the sample was determined from the mounted thermocouple and corrected for the temperature gradient between the sample and the position of the thermocouple. This gradient value has been measured independently under identical conditions with an additional thermocouple mounted at the sample position. The scattering data were corrected for background, detection efficiency of the single detector cells, radially averaged, and calibrated in absolute units by a Lupolen secondary standard. The resulting absolute macroscopic cross section $\mathrm{d} \Sigma / \mathrm{d} \Omega(\mathrm{Q})$ in units of $\mathrm{cm}^{-1}$ is related to the structure factor $\mathrm{S}(\mathrm{Q})$ according to $\mathrm{d} \Sigma / \mathrm{d} \Omega(\mathrm{Q})$ $=\mathrm{S}(\mathrm{Q}) \Delta \rho^{2} / \mathrm{N}_{\mathrm{A}}$, where $\Delta \rho$ is the difference of the coherent scattering length densities of the polymer components $\sum \mathrm{c}_{\mathrm{i}} \mathrm{b}_{\mathrm{i}} /$ $\Omega_{\mathrm{i}}$ whose numerical values are given in Tables 2 and 3 and $\mathrm{N}_{\mathrm{A}}$ is the Avogadro number. The scattering experiments were performed in the momentum transfer range of $7 \times 10^{-3} \leq \mathrm{Q}$ $\left(\AA^{-1}\right) \leq 0.1$ using the settings of $7 \AA$ neutron wavelength and the detector-to-sample distances of 2 and $6 \mathrm{~m}$ with the corresponding collimation distances. In the data analysis the instrumental resolution was taken into account according to the usual procedure. ${ }^{39}$

Different polymer melts were investigated: a polymer blend and a diblock copolymer of poly(ethylethylene) (PEE) and poly(dimethylsiloxane) (PDMS) and two diblock copolymers of poly(ethylenepropylene) (PEP) and PDMS with different molecular weight. Their characteristic parameters are given in Tables 1-3. The homopolymers and the diblock copolymers were all synthesized by anionic polymerization followed by catalytic hydrogenation (using deuterium gas). ${ }^{40}$ Their measured densities gave consistent results with an equal degree deuteration according to the chemical formula $\mathrm{C}_{4} \mathrm{D}_{2.8} \mathrm{H}_{5.2 .}{ }^{41}$ The blend and the diblock systems are symmetric with respect to their molecular weight and composition. Size exclusion chromatography (SEC) was not conducted on the first block of the diblock. However, from SEC on corresponding shorter homopolymers of PEE and PDMS we expect the polydispersity to be mostly 
Table 3. Sample Characteristics PEP-PDMS

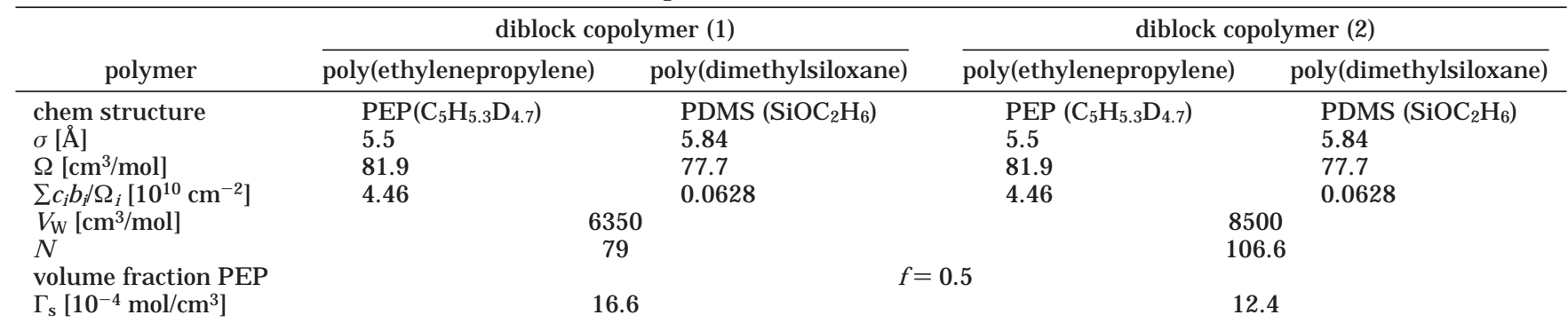

${ }^{a} \mathrm{f}$ was determined by ${ }^{13} \mathrm{C}$ and the degree of deuteration by ${ }^{1} \mathrm{H} N \mathrm{NR}$ spectroscopy. $\mathrm{V}_{\mathrm{n}}$ is the number-averaged molecular volume.

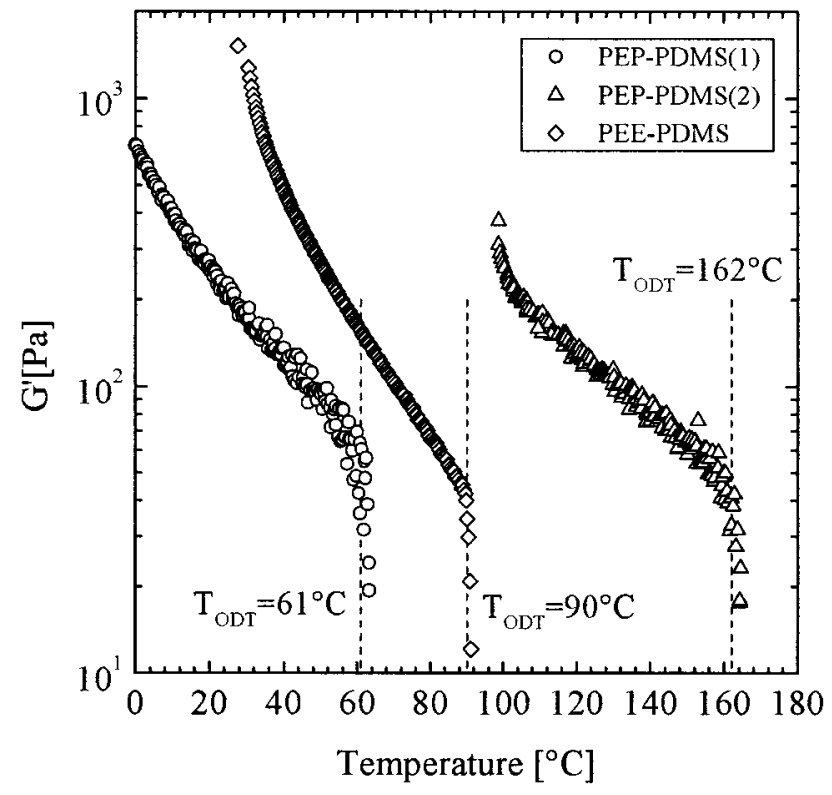

Figure 1. Elastic dynamic modulus vs temperature for the three diblock copolymers. The ordering temperatures are consistently larger by about $5 \mathrm{~K}$ as those obtained from the SANS experiments in Figure 6a probably because of temperature gradient effects.

due to the PDMS block. For a PEE with $\mathrm{V}_{\mathrm{w}}=2.07 \times 10^{3} \mathrm{~cm}^{3} /$ mol and a PDMS with $\mathrm{V}_{\mathrm{w}}=2.33 \times 10^{3} \mathrm{~cm}^{3} / \mathrm{mol}$, a polydispersity of respectively $\mathrm{V}_{\mathrm{w}} \mathrm{N}_{\mathrm{n}}=1.06$ and $\mathrm{V}_{\mathrm{w}} \mathrm{N}_{\mathrm{n}}=1.31$ was found; a block copolymer of these two species would have $V_{\mathrm{w}} N_{\mathrm{n}} \cong$ 1.17.

These systems are disordered at high temperature and ordered or phase decomposed at low temperature. This is seen in Figure 1 for the diblock copolymers from rheological measurements; the order-disorder temperature TODT of the diblocks is obtained from the strong decrease of the dynamical elastic modulus G' vs temperature.

\section{SANS Results}

A. Structure Factor of the Diblock Copolymers and Binary Homopolymer B lend. In Figures 2 and 3 examples of the structure factor $\mathrm{S}(\mathrm{Q})$ of the diblock copolymer PEP-PDMS(2) and the binary blend PEE/ PDMS are shown. Figure 2 shows S(Q) of the diblock copolymer at constant pressure and different temperatures (Figure 2a) and at constant temperature and different pressure (Figure 2b). Lowering the temperature always leads to an increase of the peak intensity. The strong increase and narrowing of $S(Q)$ between 160.3 and $155.8^{\circ} \mathrm{C}$ is a result of the phase transition from a disordered to a lamellar ordered state. Figure $2 \mathrm{~b}$ shows a sector around the peak position at the constant temperature of $\mathrm{T}=160.3{ }^{\circ} \mathrm{C}$ for various pressure fields between 0.1 and $200 \mathrm{MPa}$. A slight
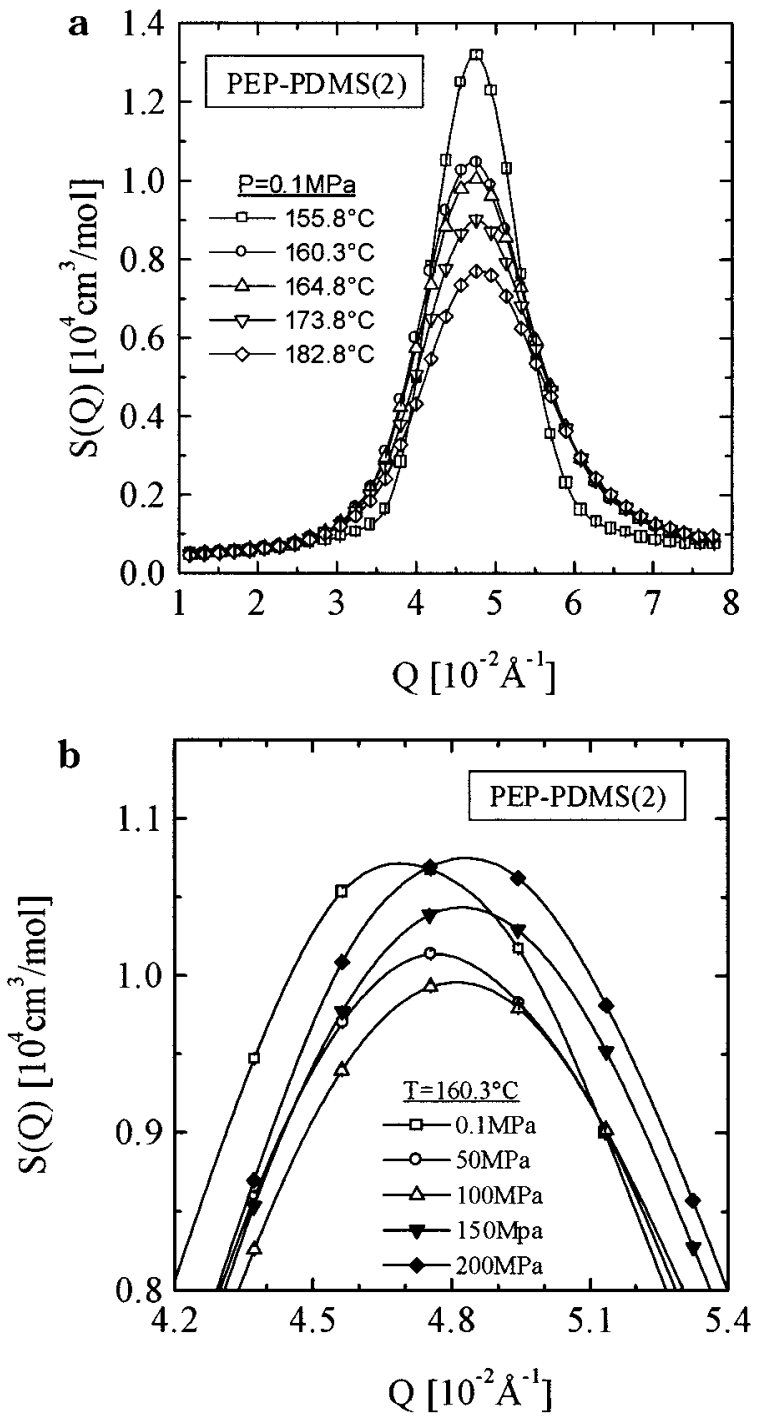

Figure 2. Structure factor of the PEP-PDMS(2) diblock copolymer at constant pressure (a) and temperature (b).

decrease and increase of $S\left(Q_{m}\right)$ is observed between 0.1 and $100 \mathrm{MPa}$ and between 100 and $200 \mathrm{MPa}$, respectively. This means that the order-disorder phase boundary first decreases and then increases with increasing pressure because thermal composition fluctuations are continuously increasing approaching the orderdisorder temperature TODT. A further observation is a continuous shift of the peak position to larger $\mathrm{Q}$ values with increasing pressure, indicating a shrinking of the polymer chains. The structure factor $\mathrm{S}(\mathrm{Q})$ delivers three characteristic parameters: the peak maximum, its halfwidth, and the peak position. The maximum $S\left(Q_{m}\right)$ is a susceptibility while the other two parameters give the 

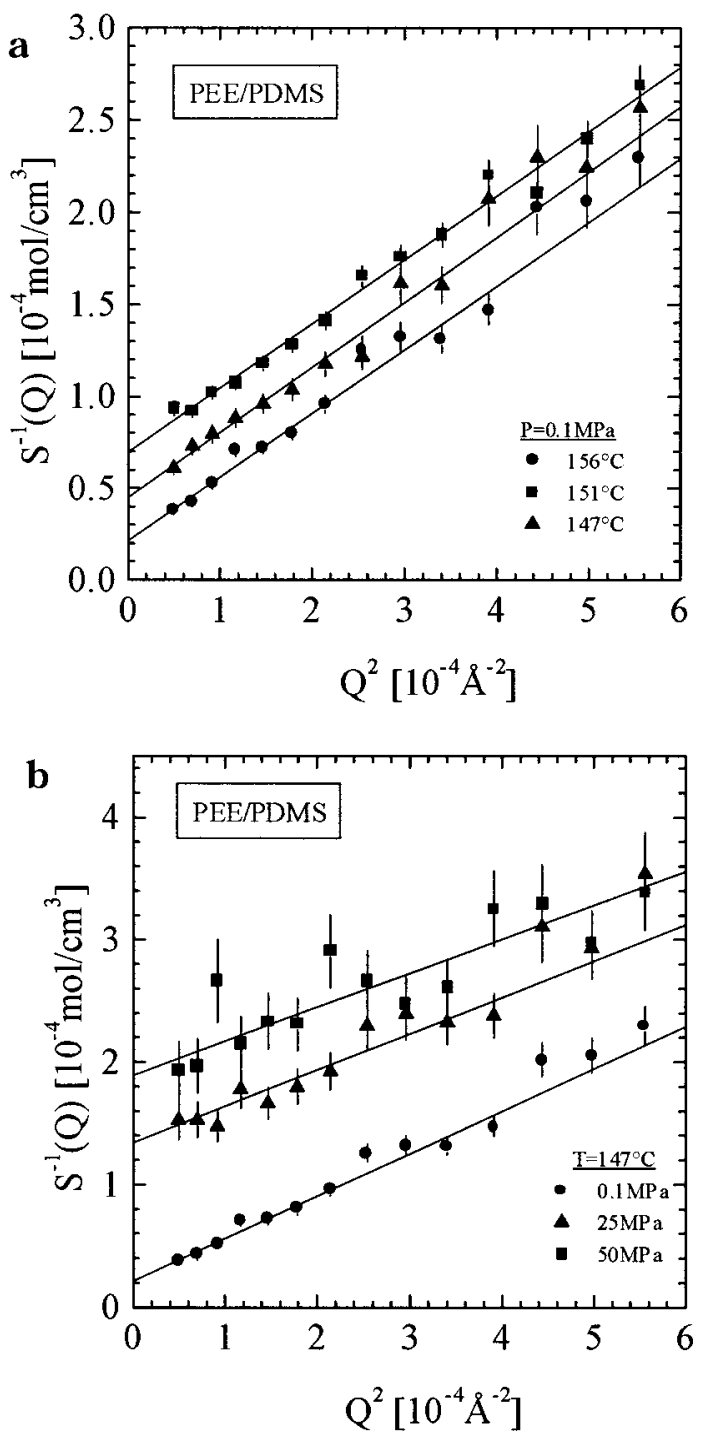

Figure 3. Structure factor of the PEE/PDMS blend in Zimm representation at constant pressure (a) and temperature (b).

correlation length of the thermal composition fluctuations and the size of the polymer chains, respectively. These parameters will be discussed in detail below.

Figure 3 shows $\mathrm{S}(\mathrm{Q})$ of the PEE/PDMS binary blend depicted in a Zimm representation. Within the statistical error bars the data follow straight lines in accordance with the predicted $S(Q)$ in eq 13 . Figure 3a represents data at constant pressure and at three different temperatures. Lowering the temperatureleads to an increase of $S(Q)$ because of approaching the phase boundary. Figure $3 \mathrm{~b}$ shows $\mathrm{S}(\mathrm{Q})$ measured at constant temperature and three different pressure fields; in the pressure range between 0.1 and $50 \mathrm{MPa}$ a decrease of $\mathrm{S}(\mathrm{Q})$ is observed. This is caused by a decrease of the critical temperature with pressure similarly to observations for the corresponding diblock copolymer in Figure $2 \mathrm{~b}$. The two parameters obtained from $S(\mathrm{Q})$, namely, the susceptibility $\mathrm{S}(0)$ and the slope $A$ according to eqs 13 and 14, will be discussed below.

B. Susceptibility and Correlation Length. In Figure $4 a, b$ the inverse susceptibility $\mathrm{S}^{-1}\left(\mathrm{Q}_{\mathrm{m}}\right)$ of two diblock copolymers have been plotted vs inverse temperature $(1 / T)$ for various pressure fields. The stepwise decrease of $\mathrm{S}^{-1}\left(\mathrm{Q}_{\mathrm{m}}\right)$ at the order-disorder temperature TODT indicates an order-disorder phase transition of
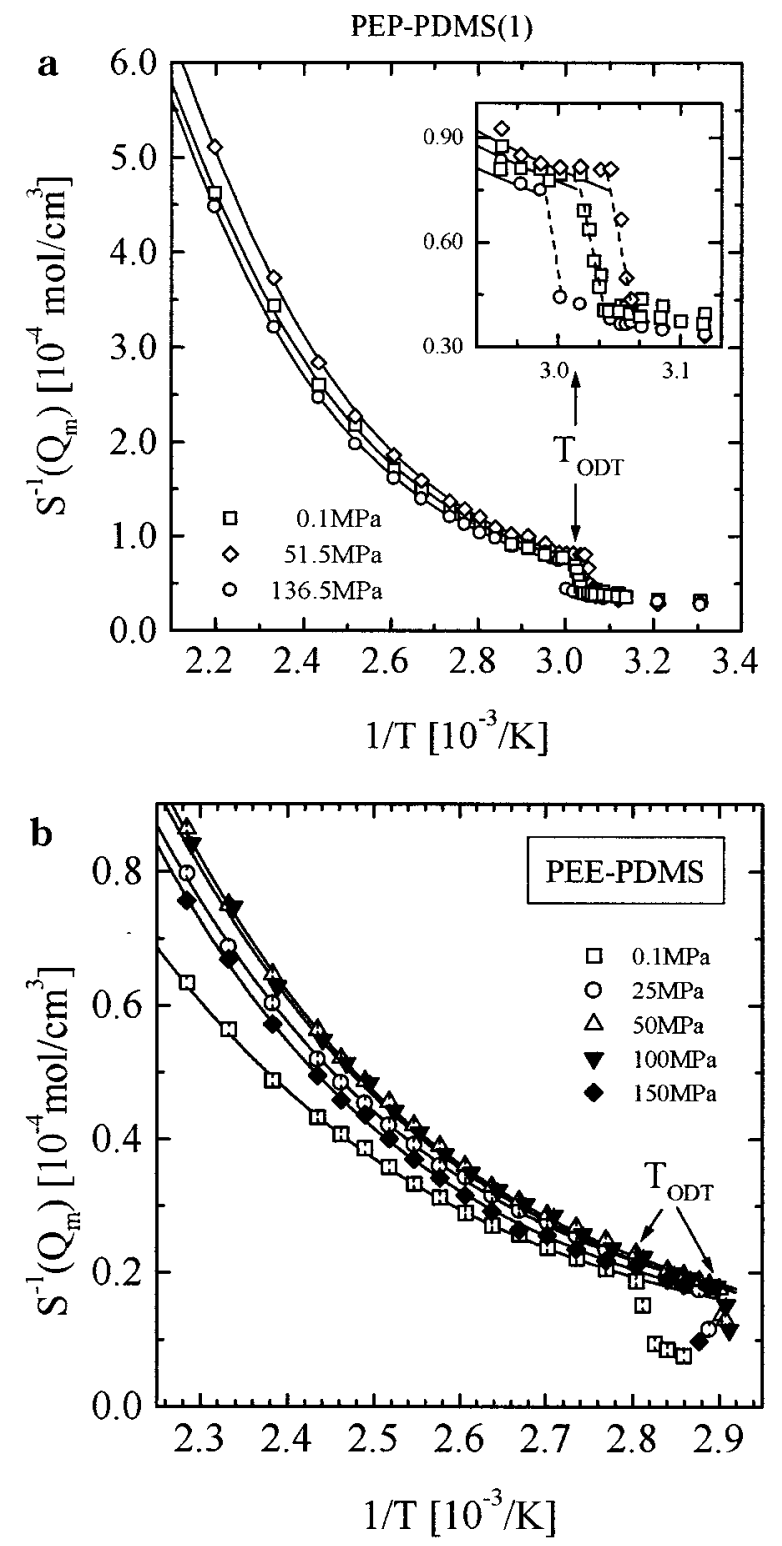

Figure 4. I nverse suscepti bility of two diblock copolymers vs inverse temperature at varying pressure fiel ds. The stepwise change of the susceptibility is clearly observed, indicating a first-order transition to a lamellar ordered phase. The solid lines represent the fitted expression of the FredricksonHelfand theory from which the Flory-Huggins interaction parameter and the Ginzburg number were evaluated.

first order. Increasing the pressure field leads first to a decrease and then to an increase of T ODT as cl early seen in the inset of Figure 4a, in agreement with the observed pressure-dependent degree of thermal fluctuations in Figure 2b. The solid lines represent a fit of the susceptibility with the Fredrickson-Helfand theory according to eqs 10 and 11 . The fitted curves describe the experimental data very well; from this fit the FloryHuggins parameter and the Ginzburg number were evaluated in accordance with eq 11.

The increase of the thermal fluctuations is accompanied by an increase of their correlation length being inversely proportional to the half-width $\Delta \mathrm{Q}_{\mathrm{m}}$ of $\mathrm{S}(\mathrm{Q})$; $\Delta \mathrm{Q}_{\mathrm{m}}$, as obtained by fitting the data to the Leibler function and calculating the corresponding widths at half-maximum value, is plotted for various pressure fields in Figure $5 a, b$ vs $1 / T$. Qualitatively, the same behavior is observed as for the susceptibility. 

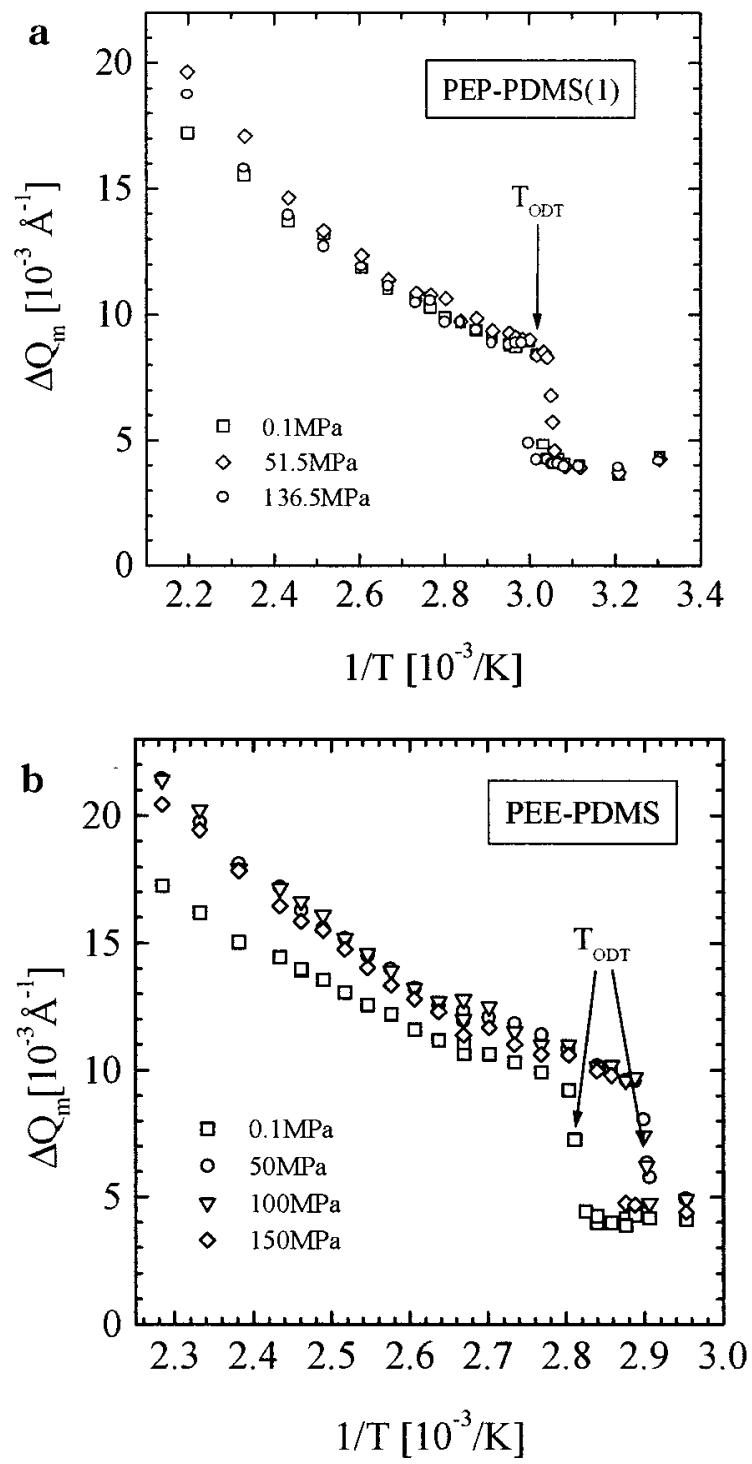

Figure 5. Full half-width vs inverse temperature of the same sample as in Figure 4. A qualitatively similar behavior as for the susceptibility is observed.

\section{Analysis and Discussion of the SANS Results}

A. Phase Diagram. The phase diagrams of all three diblock copolymers have been plotted in Figure 6a. The order-disorder phase transition temperatures are shown vs pressure as they were obtained from the stepwise change of the susceptibility. The solid lines are polynomial fits and serve as a guide for the eye. The polymers are ordered at low temperatures and disordered at high temperatures, and in all three cases the phase boundary first decreases and then increases with pressure. The transition temperatures of the two PEP-PDMS differ by about $100 \mathrm{~K}$ because of the larger molar volume of sample 2 leading to a smaller configurational entropy (see also Table 4).

In Figure $6 \mathrm{~b}$ the corresponding phase diagram of the near critical PEE/PDMS blend vs pressure is shown. The spinodal temperature was determined from the extrapolated inverse susceptibility $\mathrm{S}^{-1}(0)=0$ according to eq 14. The blend is homogeneously mixed at high temperature and phase separated at low temperature. The pressure dependence of the phase boundary is qualitatively similar to those of the diblock copolymer;
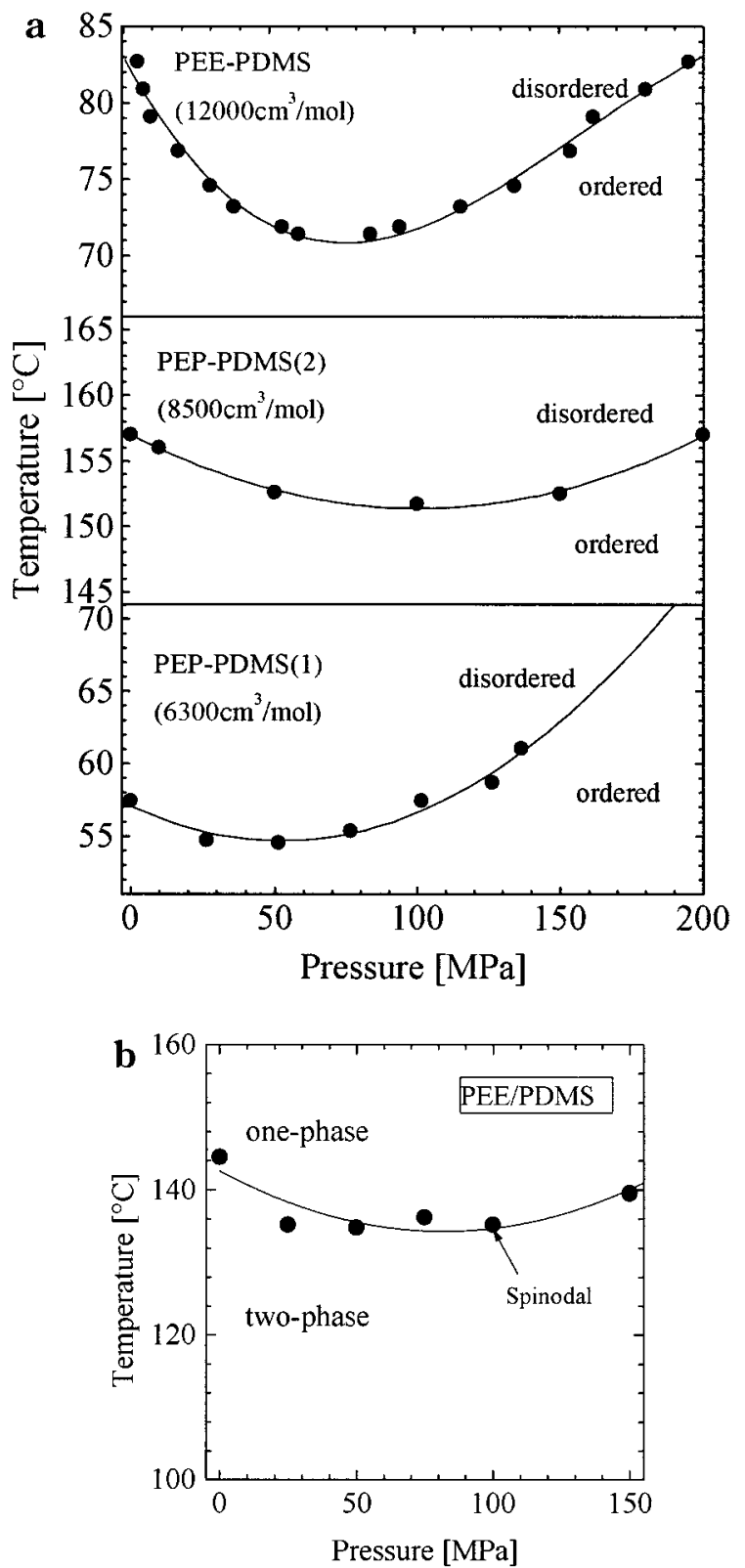

Figure 6. Phase diagrams of the diblock copolymers (a) and the blend (b). In all cases an abnormal pressure-induced decrease of the phase boundary is observed. The different transition temperatures of the PEP-PDMS diblock copolymers are caused by the different molar volumes. The relatively low transition temperature of the PE E-PDMS diblock copolymer is related to a relatively small Flory-Huggins parameter supporting a better compatibility of the two blocks.

the phase boundary first decreases and then increases with pressure.

A characteristic similarity of all studied samples is their abnormal pressure dependence of the phase boundary at the lower pressure fields showing a decrease of the phase boundary. A similar behavior was found in the binary blend PEMS/PDMS 22 and in the diblock copol ymers PEP-PEE, 18 PS-PI, 20 and PS-PMPS. ${ }^{20}$ To better understand the origin of this behavior, we have to further analyze the FH parameter and the Ginzburg number.

B. Flory-Huggins Parameter. Figure 7 shows the FH parameter $\Gamma$ vs $1 / T$ of the PEP-PDMS(1) samples for three pressures as evaluated from the FredricksonHelfand theory (eqs 10 and 11). For pressure fields 
Table 4. Thermodynamical Parameters from Experiment at $0.1 \mathrm{MPa}^{\mathrm{a}}$

\begin{tabular}{|c|c|c|c|c|}
\hline sample & $\begin{array}{l}\text { PEE/PDMS } \\
\text { (blend) }\end{array}$ & $\begin{array}{l}\text { PEE-PDMS } \\
\text { (diblock) }\end{array}$ & $\begin{array}{l}\text { PEP- } \\
\text { PDMS (1) }\end{array}$ & $\begin{array}{c}\text { PEP- } \\
\text { PDMS (2) }\end{array}$ \\
\hline $\begin{array}{l}\mathrm{T}_{\mathrm{B} ;} ; \mathrm{T}_{\mathrm{ODT}} \\
{\left[{ }^{\circ} \mathrm{C}\right]}\end{array}$ & - & 83 & 57.4 & 157 \\
\hline $\mathrm{T}_{\mathrm{S}}^{\mathrm{MF}}\left[{ }^{\circ} \mathrm{C}\right]$ & $142 \pm 3$ & - & - & - \\
\hline $\mathrm{Gi}$ & - & $1.6 \pm 0.4$ & $6.1 \pm 0.5$ & $5.4 \pm 0.5$ \\
\hline $\begin{array}{l}\Gamma_{\mathrm{h}} \\
{\left[\mathrm{mol} \mathrm{K} / \mathrm{cm}^{3}\right]}\end{array}$ & $0.8 \pm 0.2$ & $0.26 \pm 0.003$ & $1.26 \pm 0.01$ & $1.46 \pm 0.02$ \\
\hline $\begin{array}{l}\Gamma_{\sigma}\left[10^{-3}\right. \\
\left.\mathrm{mol} / \mathrm{cm}^{3}\right]\end{array}$ & $0.97 \pm 0.2$ & $0.48 \pm 0.006$ & $0.76 \pm 0.03$ & $1.15 \pm 0.05$ \\
\hline$A_{h}[K]$ & \multicolumn{2}{|c|}{6440} & \multicolumn{2}{|c|}{5250} \\
\hline $\mathrm{A}_{\sigma}$ & \multicolumn{2}{|c|}{5.9} & \multicolumn{2}{|c|}{9.7} \\
\hline$\Gamma_{\text {ODT }} \mathrm{V}$ & - & 11.8 & 19.1 & 19.1 \\
\hline
\end{tabular}

a The given error bars were obtained from statistical methods of the fitting routines including the statistical error of the experimental data.

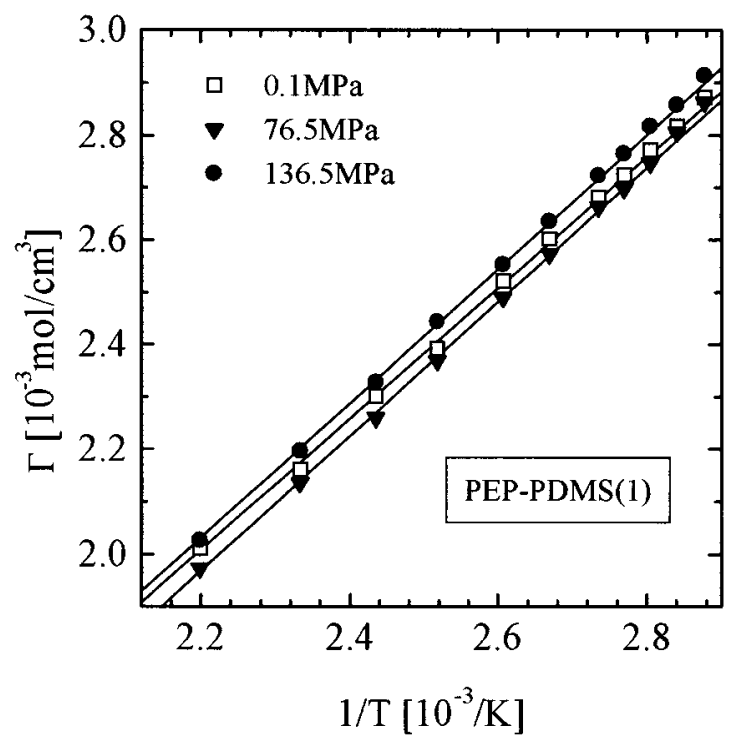

Figure 7. Flory-Huggins parameter of the diblock copolymer PEP-PDMS(1) for three pressure fields as evaluated from the susceptibility in Figure $4 a$ with the Fredrickson-Helfand theory. The obtained straight line is consistent with ansatz $\Gamma$ $=\Gamma_{\mathrm{h}} / \mathrm{T}-\Gamma_{\sigma}$.

between 0.1 and $76.5 \mathrm{MPa}$ the value of $\Gamma$ slightly decreases and then increases, showing the maximum value at $136.5 \mathrm{MPa}$. The lower $\Gamma$ means a better compatibility of the two polymer components and therefore a decrease of the phase boundary as observed in Figure 6. The FH parameter follows $\Gamma=\Gamma_{\mathrm{h}} / \mathrm{T}-\Gamma_{\sigma}$ from which the respective enthalpic and entropic terms $\Gamma_{h}$ and $\Gamma_{\sigma}$ were evaluated. These both parameters are depicted vs pressure in Figure 8a-c and in Figure 9 for the diblock copolymers and for the blend, respectively (see also Table 4). In all cases the enthalpic and the entropic term first increases and then decreases with pressure. The maximum values are found at around 100 $\mathrm{MPa}$. The absolute value of $\Gamma$ for the PEE-PDMS sample is about 5 time smaller than for the PEP-PDMS sample, indicating a better compatibility. This is also seen from the phase diagram in Figure 6 where the PEE -PDMS sample with a twice as large mol ar volume leads to a phase boundary of slightly higher temperature than the PEP-PDMS(1) sample. Quite generally, a decrease of the phase transition temperature reflects an overall lower FH interaction parameter, which can be caused by a dominating decrease or increase of the enthalpic or entropic term, respectively. Since both FH
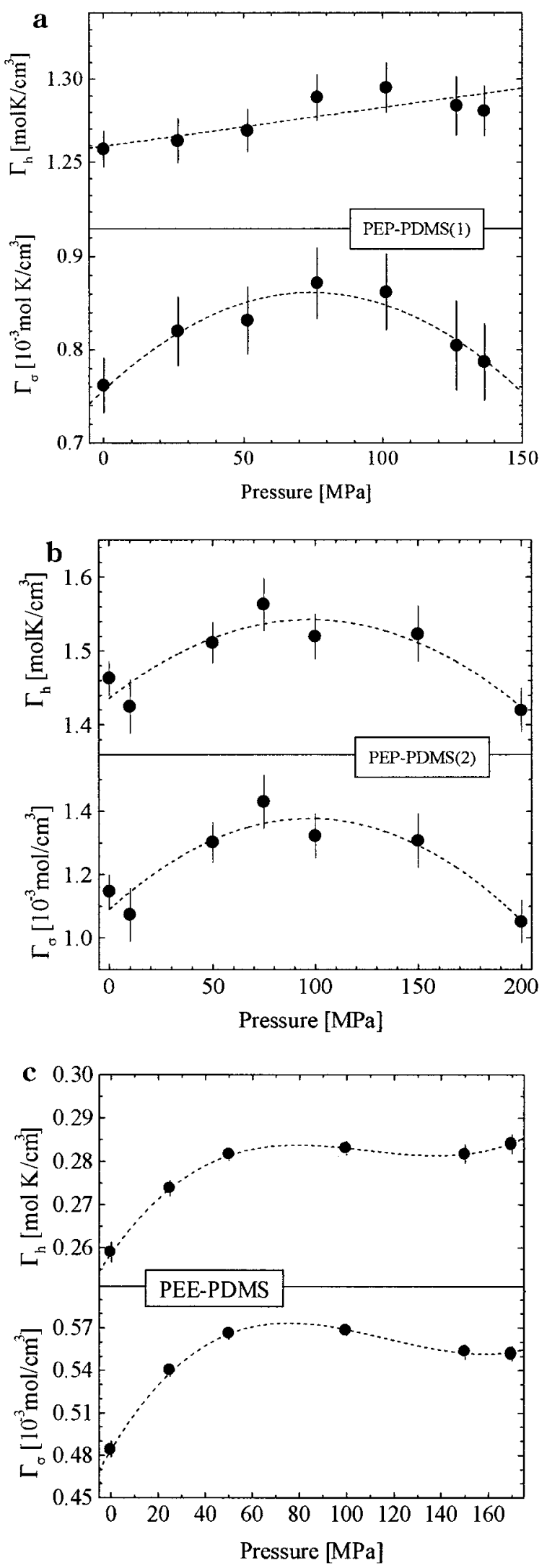

Figure 8. Enthal pic and entropic terms of the Flory-Huggins parameter of the diblock copolymers as evaluated from data plotted in Figure 7. In low-pressure fiel ds al ways a pressure induced increase of both parameters is observed.

terms according to the data analysis change (are increasing) with pressure in the same direction, the entropic term must be the dominating one. 


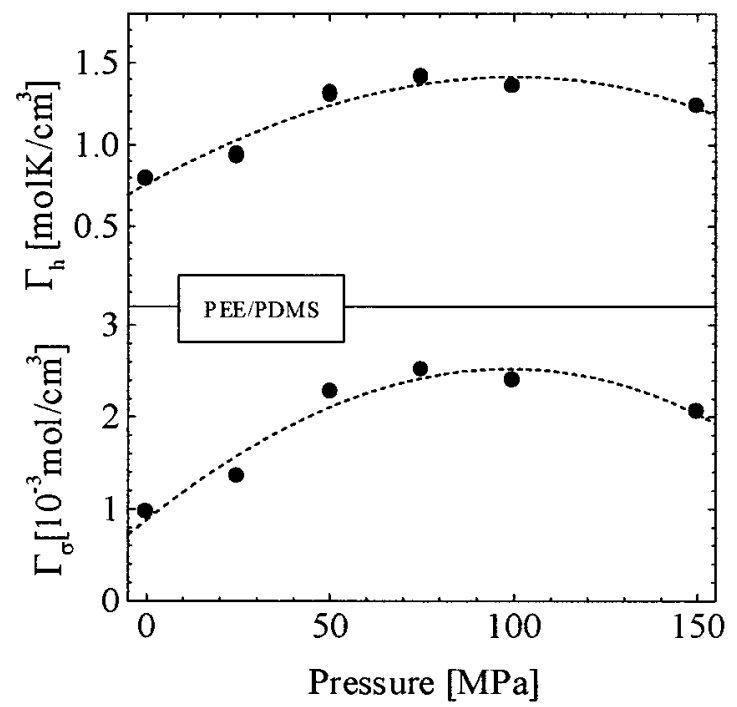

Figure 9. Enthal pic and entropic terms of the F lory-Huggins parameter of the blend. The pressure-dependent changes are qualitatively consistent with those of the diblock copolymers in Figure 8.

The relationship of the pressure dependence of the phase boundary with thermodynamic parameters is described by the Clausius-Clapeyron equation in eqs 1 and 5 . As the phase boundaries and the FH parameter are independently obtained from the SANS data, namely, the transition temperature from a stepwise change of the susceptibility and/or the correl ation length and the $\mathrm{FH}$ parameter from the temperature dependence of the susceptibility within the whole disordered regime, a cross-check of the $\mathrm{FH}$ parameter by the ClausiusClapeyron equation according to eq 5 is possible. The change of the phase boundary with pressure is determined by two effects, namely, by the change of the $\mathrm{FH}$ parameter shown in Figures 8 and 9 and by the change of the degree of thermal fluctuations expressed by a pressure-dependent Ginzburg parameter (see eq 12). In other studies a decrease of the Ginzburg number with pressure was observed in polymer blends and diblock copolymers. ${ }^{13,15}$ In the present diblock samples we observed a constant Ginzburg number within the error bars. For the present blend the SANS measurements were performed with too large temperature steps near the phase boundary in order to observe deviations from the mean field behavi or and being able to determine the corresponding Ginzburg number. So, in accordance with eq 5 we interpret the pressure-induced changes of the phase boundaries as caused al one by the $\mathrm{FH}$ parameter plotted in Figures 8 and 9. The change of the phase boundary with pressure is given in Figure 10a,b for the diblocks and the blend. The dashed lines represent values directly evaluated from the fitted phase boundary in Figures 8 and 9 while the solid lines were evaluated from the $\mathrm{FH}$ parameter in accordance with the Clausius-Clapeyron equation in eq 5 . The agreement between both curves is satisfying and therefore, in particular, gives confidence to the Fredrickson-Helfand formalism for dibl ock copolymers. It, furthermore, gives in terms of the $\mathrm{FH}$ parameter an explanation of the observed abnormal pressure dependence of the phase transition temperature.

A further question is related to the properties of the $\mathrm{FH}$ parameter of two polymer chains being in one case mixed as a blend and in the other case connected as a diblock copolymer. Implicitly, the theoretical approaches
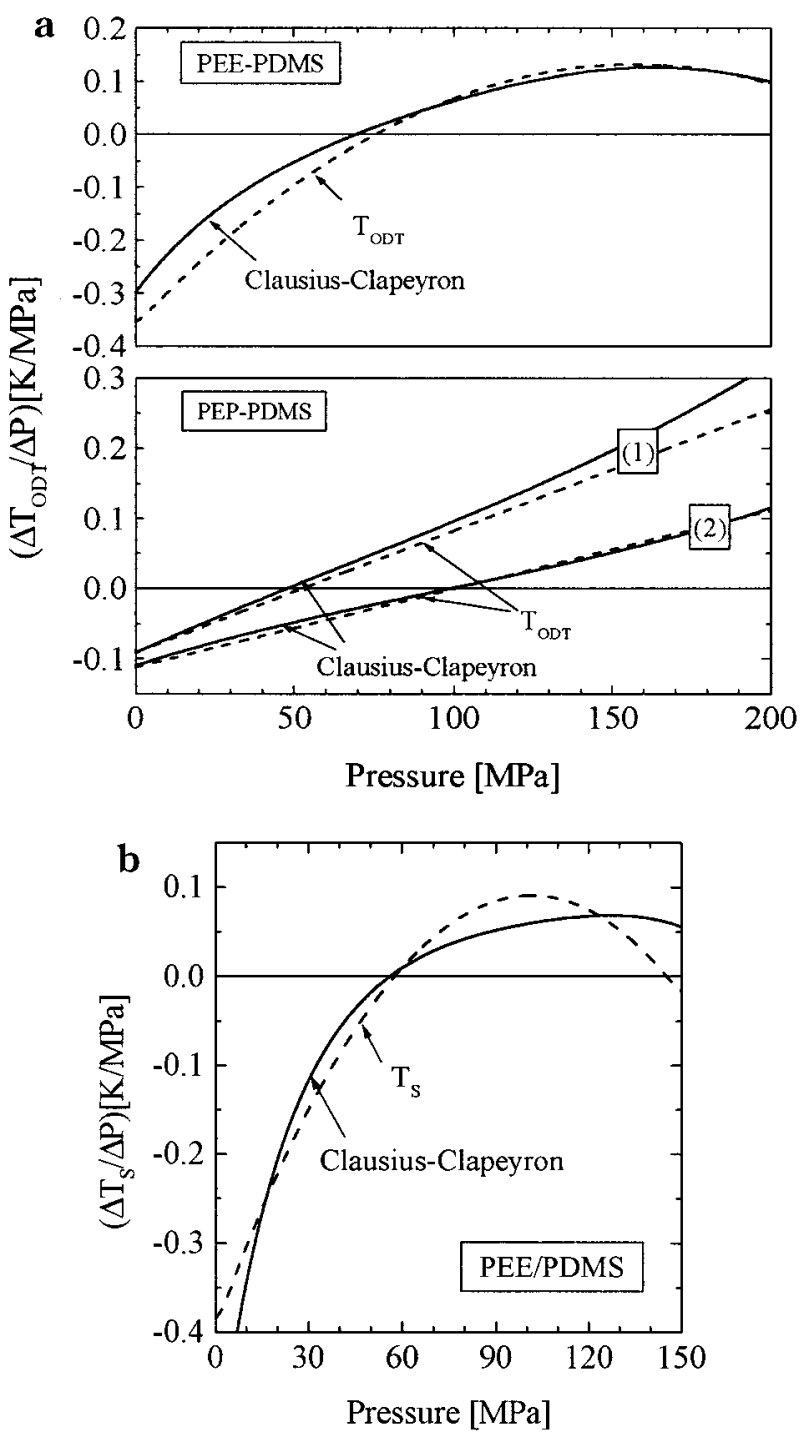

Figure 10. Pressure-induced changes of the transition temperatures of the diblock copolymers (a) and blend (b). The dashed line show temperature changes directly obtained from the phase boundaries in Figure 6 while the solid lines have been evaluated from the Flory-Huggins parameter in Figures 8 and 9 using the Clausius-Clapeyron equation.

assume that the $\mathrm{FH}$ parameter depends only on the monomers and on end effects which may be visible only for small molecular chains. In eq 15 a relationship of the FH parameters between blends and diblock copolymers has been proposed by Freed and Dudowicz. ${ }^{30}$ The contact between the two blocks gives an additional contribution proportional to $1 / \mathrm{N}$. The corresponding coefficients of the entropic and enthalpic terms as obtained from experiment are plotted in Figure 11 vs pressure for the PEP-PDMS and PEE-PDMS systems of the blend and diblock copolymers together with polynominal fits giving a guide for the eye (see also Table 4). The enthal pic and entropic coefficients A could be analyzed as for each system two samples were studied: for PEP-PDMS the two diblock copolymers of different molar volumes and for PEE-PDMS a diblock and a blend system. The more compatible PEE-PDMS mixture shows slightly stronger pressure dependence with absolute numbers that are of the same order of magnitude. A similar analysis of a PB-PS blend and diblock copolymer ${ }^{15}$ and on the PEP-PEE diblock copolymer ${ }^{18}$ shows coefficients of similar sizes. 


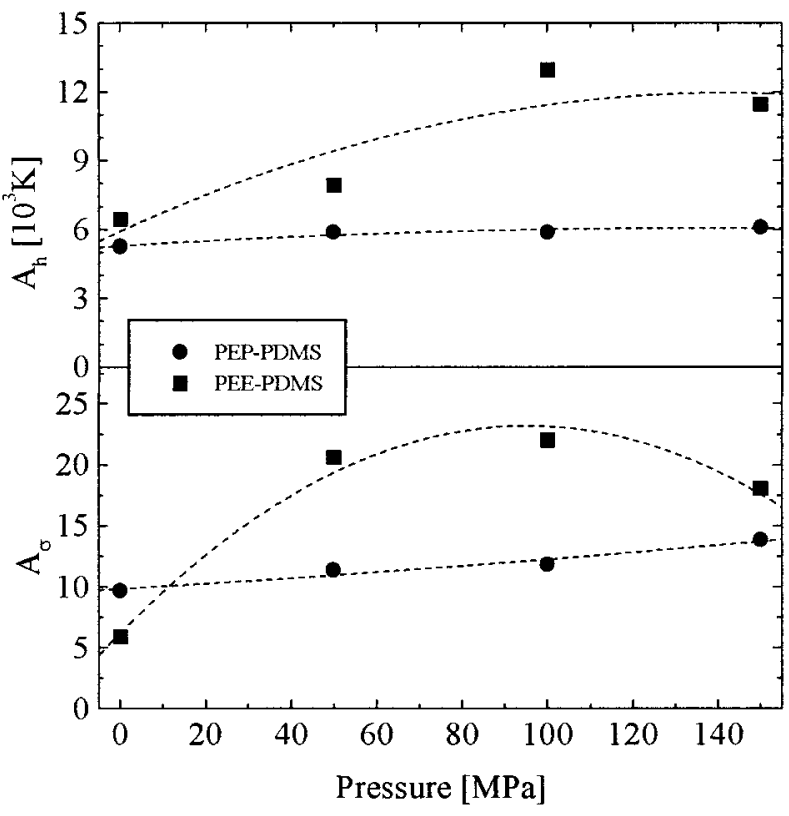

Figure 11. Parameters of the enthal pic and entropic FloryHuggins parameter in eq 15 vs pressure describing a relationship between the Flory-Huggins parameter of diblock copolymers and polymer blends as proposed by LCT calculations.

The FH parameter of all samples irrespective of being a diblock copolymer or a binary blend shows qualitatively the same behavior, namely, first an increase and then a slight decrease of both $\Gamma_{\mathrm{h}}$ and $\Gamma_{\sigma}$ with pressure. In the pressure range of increasing $\Gamma_{\mathrm{h}}$ and $\Gamma_{\sigma}$ a negative change of the phase boundary is observed. The Clausius-Clapeyron equation gives consistent results, namely, a dominant contribution of the entropic $\Gamma_{\sigma}$ term. No change of the Ginzburg number with pressure was observed which means that the change of the phase boundary with pressure is solely determined by changes in the $\mathrm{FH}$ parameter. The $\mathrm{FH}$ parameter of polymer systems showing abnormal changes of the phase boundary as the PEMS/PDMS blend in ref 22 shows qualitatively the same behavior as in the presented samples while an other polymer system shows a different behavior: in the PEE - PEP diblock copolymer increasing pressure leads to a decreasing $\Gamma_{\mathrm{h}}$, while $\Gamma_{\sigma}$ remains constant. ${ }^{18}$ It is characteristic that the two blocks of this sample are rather compatible as they have only a small FH parameter; $\Gamma_{\mathrm{h}}$ being about 4 times smaller than the corresponding PEE-PDMS value given in Figure 8c, and $\Gamma_{\sigma}$ is equal to $-1.8 \times 10^{-5} \mathrm{~mol} / \mathrm{cm}^{3} .21$

C. Ginzburg Number. The Ginzburg number of these diblock copolymer samples shows no pressure dependence within the experimental uncertainty as shown for the PEP-PDMS(1) sample in Figure 12a (see also Table 4). This is in contrast to observations of the PB-PS system where a clear decrease of $G i$ with $P$ was observed. ${ }^{15}$ As now a collection of Ginzburg numbers from several diblock copolymers with different molar volume are available, we have plotted these numbers in Figure $12 \mathrm{~b}$ vs the molar volume in a doublelogarithmic representation. These Ginzburg numbers comprise data obtained from SANS experiments of samples from the present work as well as data from refs 15, 18, and 23. The PEP-PEE (ref 18) and PB-PI (ref 23) diblock copolymers of relatively large molar volumes could be studied because of the al ready mentioned good compatibility of the PEP and PEE blocks and of the ordering transition at high temperatures, respectively.
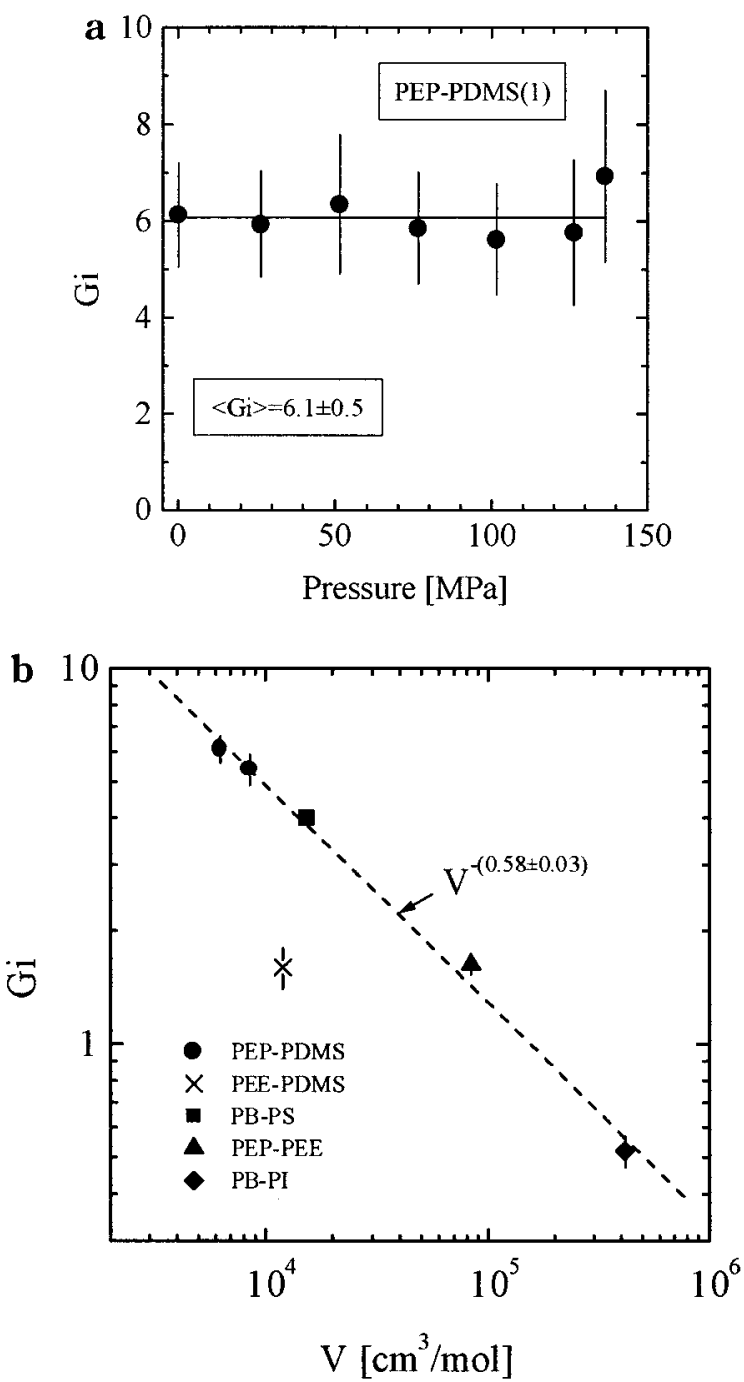

Figure 12. (a) Example of a Ginzburg number vs pressure as evaluated from the Fredrickson-Helfand theory. $\mathrm{Gi}$ is constant within the error bars. (b) Ginzburg number vs mol ar volume from different diblock copolymers in double-logarithmic scale. The scaling exponent is near 0.5 as proposed (see text bel ow eq 11). The deviation of Gi from the PEE-PDMS sample is in contrast to a universal law.

The Ginzburg number determined for molar volumes over 2 orders of magnitude follow, excluding the value of the PEE-PDMS sample, a near perfect scaling law with an exponent of -0.58 , which is very near the theoretically predicted universal scaling behavior with slope -0.5 . The PEE-PDMS sample shows roughly a 3 times smaller $\mathrm{Gi}$, indicating that there anyway are limitations for universality.

D. Polymer Chain Conformation and Compressibility. The peak position is the third parameter obtained from S(Q). It will be discussed separately as it gives more microscopic information about the size of the diblock copolymer; within the Leibler mean field theory and the Fredrickson-H el fand theory the radius of gyration follows the relationship $\mathrm{R}_{g} \mathrm{Q}_{m}=1.945$; e.g., assuming $\mathrm{R}_{\mathrm{g}}$ constant, $\mathrm{Q}_{\mathrm{m}}$ is expected to be constant with temperature and pressure. 7,9 Experiments, $31,34,35$ extensions beyond the Fredrickson-Helfand theory, 32,33 and computer simulations, ${ }^{36}$ however, have shown that the polymer is stretching if continuously approaching the ordering phase transition. The simulation studies have also shown that $Q_{m}$ does not only represent pure 

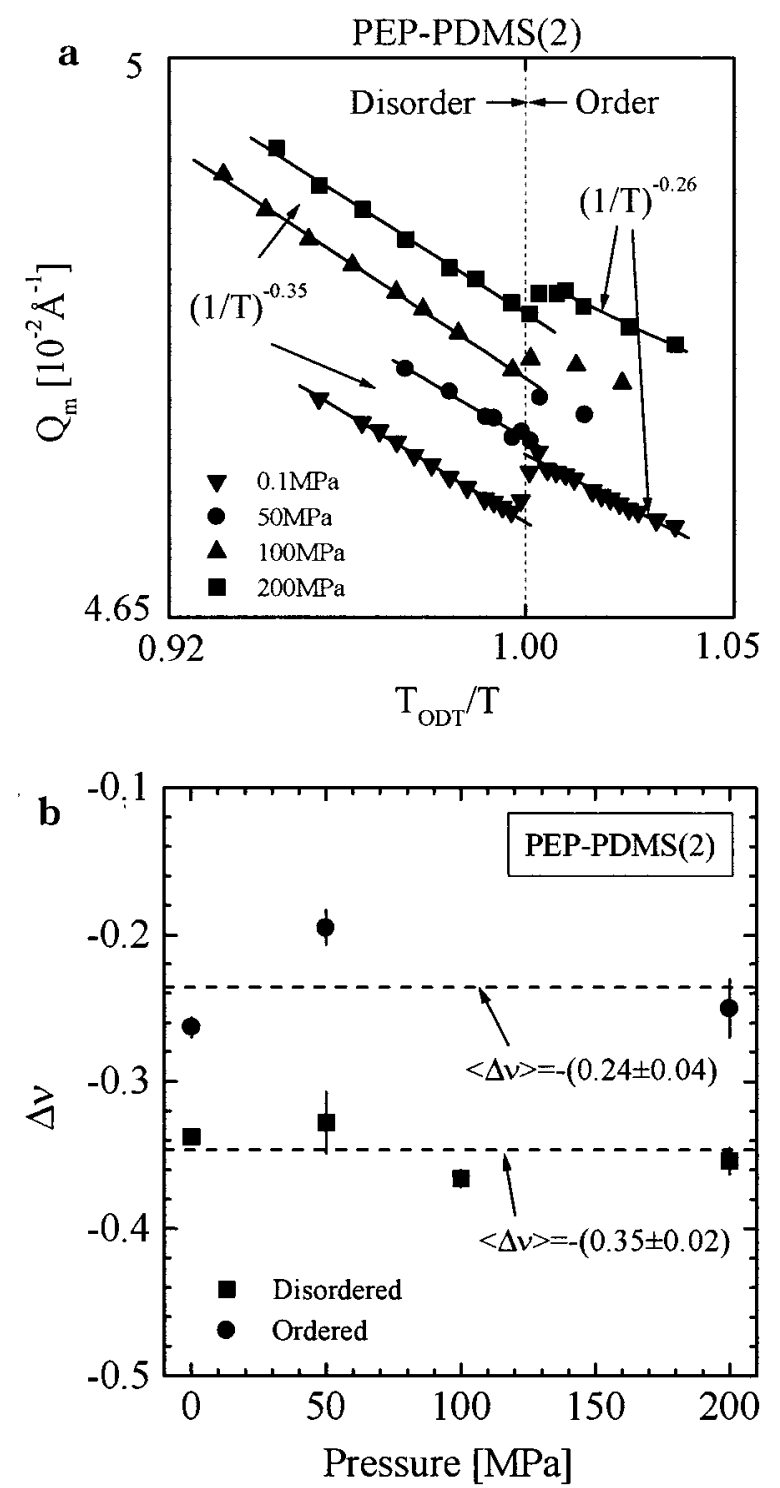

Figure 13. Peak position of the structure factor vs inverse temperature in double-logarithmic scale (a). A scaling behavior is observed in the disordered and ordered regimes. The corresponding exponents in (b) give within the error bars constant numbers. A stepwise decrease of the chain size is always observed at the transition temperature.

chain properties but al so reflects cooperative effects. In Figure $13 a Q_{m}$ of the PEP-PDMS(2) sample is plotted vs the inverse temperature for various pressure fields in a double-logarithmic scale; the temperature has been normalized with the order-disorder temperature. Two scaling laws are observed, separated by the phase boundary. The corresponding exponents are plotted in Figure 13b; in the disor dered regime $\mathrm{Q}_{m}$ decreases with temperature with an exponent of -0.35 and in the ordered regime with slightly smaller absolute exponent which always means a stretching of the polymer confirmation. These findings are in good quantitative agreement with simulation calculations, finding a maximum exponent of $\Delta v \cong-0.24$ near the transition temperature. ${ }^{36}$ The simulations were performed over a much larger temperature range and therefore show a crossover to Gaussian coil behavior with a zero exponent at high temperatures. Within error bars the exponents in Figure 13b are constant while the coil size itself is appreciably decreasing with pressure.
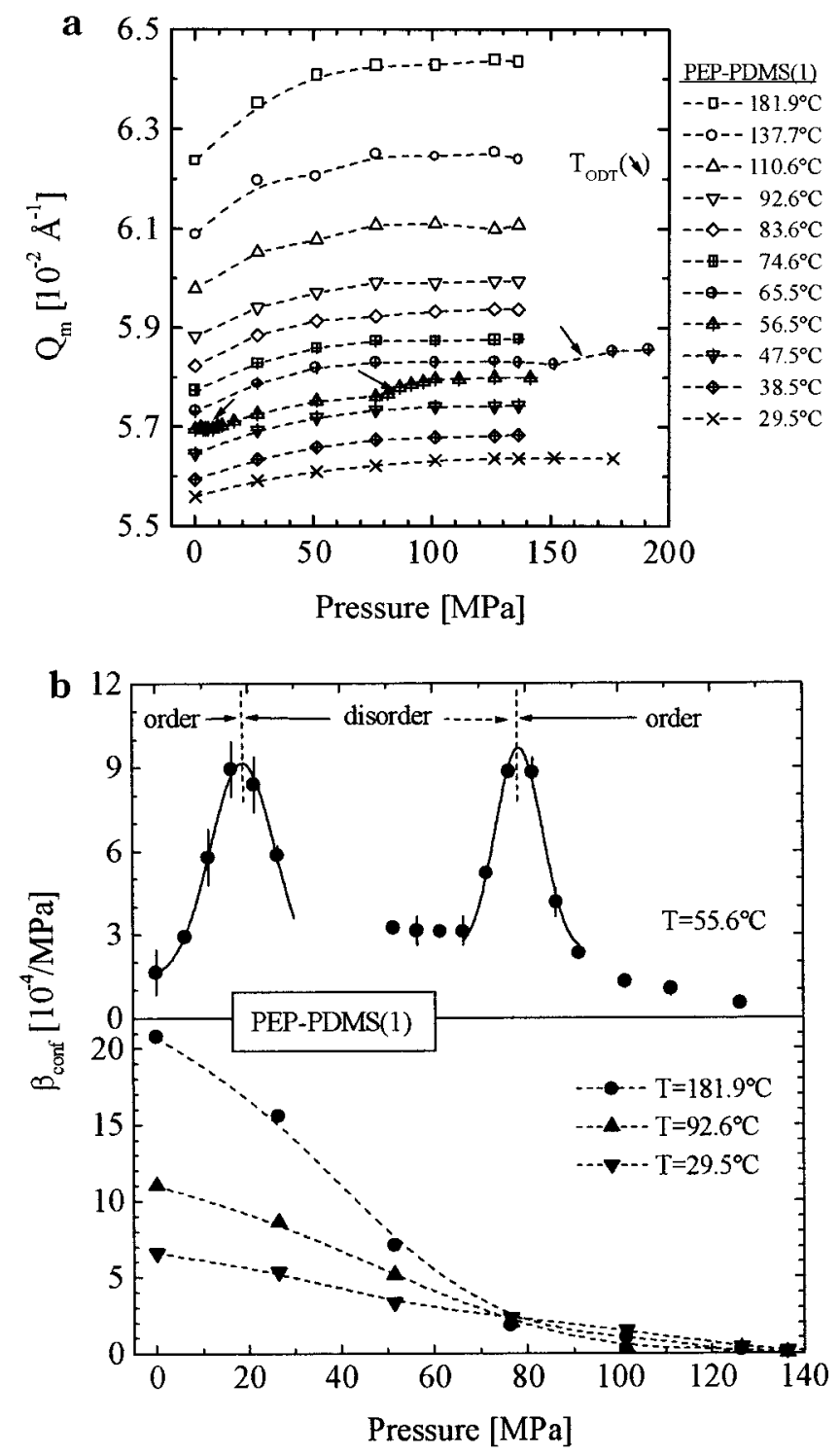

Figure 14. (a) Peak position vs pressure for the PEP-PDMS(1) diblock copolymer for different temperatures. The compressibility according to eq 16 in (b) shows always a decrease a higher pressure fields. At the ODT transition a hump is observed.

At the transition temperature a stepwise decrease of the size of the polymer coil is observed in all measurements presented in this paper. A discontinuous change in the coil size has, according to calculations by Stepanow beyond the random phase approximation, ${ }^{33}$ been predicted at the TODT, however, with a stepwise increase of the coil size. A stepwise decrease of the coil size was also observed by Ogawa et al., however, only for an asymmetric diblock copolymer forming cylindrical microdomains. 35

In Figures $14 a$ and $15 a Q_{m}$ has been depicted vs pressure for the samples PEP-PDMS(1) and PEEPDMS. In all cases the coil radius decreases with increasing pressure and temperature. From the data in these two plots the compressibility of the chains has been evaluated according to eq 16 and is plotted in Figures $14 \mathrm{~b}$ and $15 \mathrm{~b}$. With increasing pressure and decreasing temperature the compressibility is declining. Quite remarkable is the observed hump in the compressibility at the ordering transitions, $\mathrm{T}_{\mathrm{ODT}}$, which is of course reflecting the directly observed stepwise 

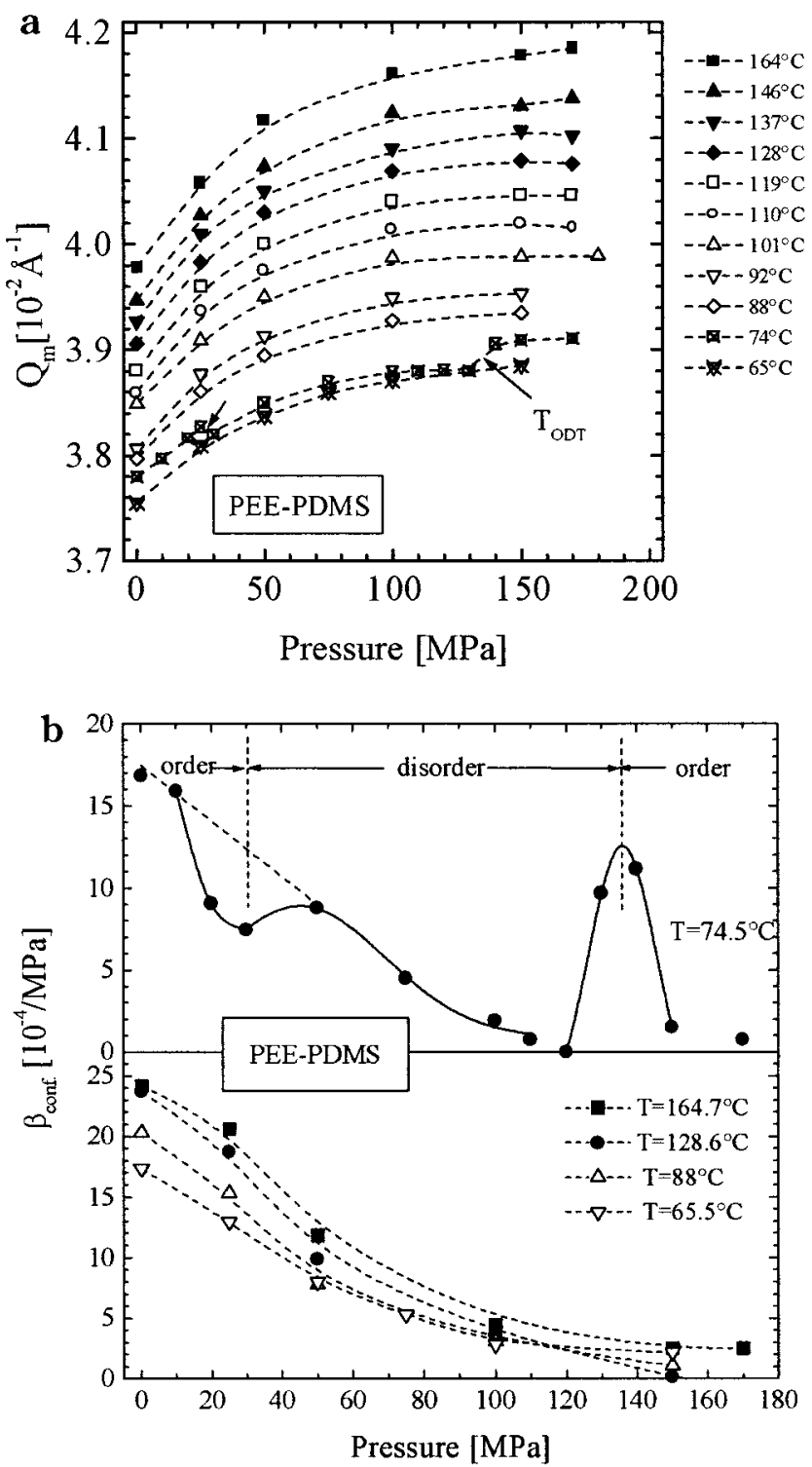

Figure 15. Same as in Figure 14 but for the PEE-PDMS diblock copolymer. A qualitatively similar behavior is found.

decrease of the chain size at TODT, as shown in Figure $13 a, 14 a$, and 15a.

\section{Summary and Conclusions}

We have studied thermal composition fluctuations in the disordered phase of several binary polymer melts as a function of temperature and pressure. We studied two PEP-PDMS diblock copolymers of different molar volumes, a PEE-PDMS diblock copolymer and a PEE/ PDMS binary blend. In all cases we had symmetric diblock copolymers $(\phi \cong 0.5)$ and a blend near its critical composition ( $\phi \cong 0.5$ ). We determined as a function of pressure the phase boundaries of respectively microphase separation into lamellar ordered structure and macrophase decomposition, the F lory-Huggins interaction parameters, and for the diblock copolymers the Ginzburg number and the chain compressibility. As shown in Figure 6, it is found that for all samples the phase boundary abnormally decreases at low-pressure fields and increases at higher applied pressures. An increase of the phase boundary with pressure is expected as a result of the reduction of the free volume and thereby of entropic contributions by pressure. Such behavior has been observed in many binary polymer blends and in some diblock copolymers. The increasing phase boundary was also predicted from calculations based on lattice cluster theory (LCT), when the effect of compressibility was included. ${ }^{16}$ It has been discussed whether a decrease of the phase boundary with pressure might be the normal case for diblock copolymers, since this for some time has been the situation observed experimentally in most diblock copolymer systems. More recent SANS experiments, however, suggest that this is not the case: blends and diblock copolymers of polybutadiene (PB) and polystyrene (PS) show qualitatively the same pressure behavior. ${ }^{15}$ In the present studies we also find similar behavior of diblock and homopolymer blends. A major result of the present work is therefore to demonstrate that the pressure dependence is mainly determined by the properties of mutual interaction between the various polymer segments.

The pressure-dependent $\mathrm{FH}$ parameters plotted in Figures 7-9 were determined from the measured susceptibility in the disordered regime, analyzed on the basis of the Flory-Huggins and the FredricksonHelfand theoretical approaches for respectively blends and diblock copolymers. Both the enthalpic and the entropic terms of $\Gamma$ first increase and then decrease with pressure, consistent with the phase boundary described via the Clausius-Clapeyron equation. It is concluded that the decrease of the phase boundaries at lowpressure fields is caused by an entropic increase which dominates over the enthalpic contribution.

It is known that thermal fluctuations expressed by the degree of the Ginzburg number Gi stabilizes the disordered phase. As Gi of the present diblock copolymers is found constant with pressure (Figure 12a), the change of phase boundaries with pressure is not affected by thermal fluctuations. In Figure 12b we furthermore give a collection of $\mathrm{Gi}$ from several diblock copolymers studied by us over the recent years; a scaling behavior of $\mathrm{Gi}$ vs the molar mass is found for most systems with an exponent slightly larger than the predicted 0.5 (see subsequent text of eq 11).

Finally, we discuss a more microscopic quantity of the diblock copolymers obtained from the peak position of the structure factor $S(Q)$ which is related to the size of the polymer chains. It was found from simulation studies that this number represents a collective length originating from the cooperative behavior of many chains. ${ }^{36}$ As seen in Figure 13a for all pressure fields the chain length is stretching with decreasing temperature approaching the ordering transitions, while an increase of pressure causes shrinking chain conformation. At the TODT transition temperature a slight, but distinct, stepwise increase is observed in $\mathrm{Q}_{\mathrm{m}}$, leading to a weak singularity of the chain compressibility (Figures 14b and 15b); the corresponding shrinking of stretched polymers chains leads to an increase of the configurational entropy. The peak maximum $\mathrm{Q}_{\mathrm{m}}$ follows vs $1 / T$ a scaling behavior with an exponent of -0.33 in the disordered regime. This is quantitatively consistent with simulation studies; the stepwise change of $\mathrm{Q}_{m}$ at TODT, however, was not observed in the numerical simulations. ${ }^{36}$

Acknowledgment. This work has been supported by the European program for large-scale facilities (TMR). 


\section{References and Notes}

(1) deGennes, P. G. Scaling Concepts in Polymer Physics; Cornell University Press: Ithaca, NY, 1979.

(2) Binder, K. Adv. Polym. Sci. 1994, 112, 181.

(3) Schwahn, D.; Mortensen, K. Thermal Composition Fluctuations in Polymer Blends Studied with Small Angle Neutron Scattering. In Scattering in Polymeric and Colloidal Systems; Brown, W., Mortensen, K., Eds.; Gordon \& Breach Publishers: Langhorne, PA, 2000.

(4) Bates, F. S.; Fredrickson, G. H. Annu. Rev. Phys. Chem. 1990 $41,525$.

(5) Hamley, I. W. The Physics of Block Copolymers; Oxford Science Publications: Oxford, 1998.

(6) Brazovskiî, S. A. Sov. Phys.-J ETP 1975, 41, 85.

(7) Leibler, L. Macromolecules 1980, 13, 1602.

(8) Ohta, T.; Kawasaki, K. Macromolecules 1986, 19, 2621.

(9) Fredrickson, G. H.; Helfand, E. J . Chem. Phys. 1987, 87, 697.

(10) Fredrickson, G. H.; Helfand, E. J . Chem. Phys. 1988, 89, 5890

(11) J anssen, S.; Schwahn, D.; Mortensen, K.; Springer, T. Macromol ecules 1993, 26, 5587; 1995, 28, 2555.

(12) Hammouda, B.; Bauer, B. J . Macromol ecules 1995, 29, 4505

(13) Schwahn, D.; Schmackers, T.; Mortensen, K. Phys. Rev. E 1995, 52, R1288.

(14) Hammouda, B.; Balsara, N. P.; Lefebvre, A. A. Macromolecules 1997, 30, 5572.

(15) Frielinghaus, H.; Abbas, B.; Schwahn, D.; Willner, L. Europhys. Lett. 1998, 44, 606.

(16) Dudowicz, J .; Freed, K. F. J . Chem. Phys. 1994, 100, 4653; Macromol ecules 1995, 28, 6625.

(17) Hajduk, D. A.; Urayama, P.; Gruner, S. M.; Register, R. A.; Brister, K.; Fetters, L. J. Macromolecules 1995, 28, 7148.

(18) Frielinghaus, H.; Schwahn, D.; Mortensen, K.; Almdal, K.; Springer, T. Macromolecules 1996, 29, 3263.

(19) Kasten, H.; Stühn, B. Macromol ecules 1995, 28, 4777.

(20) Steinhoff, B.; Rüllmann, M.; Wenzel, M.; J unker, M.; Alig I.; Oser, R.; Stühn, B.; Meier, G.; Diat, O.; Bösecke, P.; Stanley, H. B. Macromolecules 1998, 31, 36.

(21) Schwahn, D.; Frielinghaus, H.; Mortensen, K.; Almdal, K. Phys. Rev. Lett. 1996, 77, 3153.

(22) Beiner, M.; Fytas, G.; Meier, G.; Kumar, S. K. Phys. Rev. Lett. 1998, 81, 594.
(23) Hasegawa, H.; Sakamoto, N.; Takeno, H.; J innai, H.; Hashimoto, T.; Schwahn, D.; Frielinghaus, H.; J anssen, S.; I mai, M.; Mortensen, K. J . Phys. Chem. Solids 1999, 60, 1307.

(24) Ma, S. K. Statistical Mechanics; World Scientific: Singapore, 1982.

(25) Sengers, J.v. In Supercritical Fluids: Fundamentals for Application; Kiran, E., Levelt Sengersk, J. M. H., Eds.; Kluwer Academic Publishers: Dordrecht, 1994.

(26) Schwahn, D.; Meier, G.; Mortensen, K.; J anssen, S. J . Phys. II 1994, 4, 837.

(27) Higgins, J . S.; Benoit, H. Polymers and Neutron Scattering; Clarendon Press: Oxford, 1994.

(28) Muthukumar, M. Macromolecules 1993, 26, 5259.

(29) Hohenberg, P. C.; Swift, J . B. Phys. Rev. E 1995, 52, 1828.

(30) Dudowicz, J .; Freed, K. Macromolecules 1993, 26, 213.

(31) Almdal, K.; Rosedale, J. H.; Bates, F. S.; Wignall, G. D.; Fredrickson, G. H. Phys. Rev. Lett. 1990, 65, 1112.

(32) Barrat, J .-L.; Fredrickson, G. H. J . Chem. Phys. 1991, 95, 1281.

(33) Stepanow, S. Macromolecules 1995, 28, 8233.

(34) Stühn, B.; Mutter, R.; Albrecht, T. Europhys. Lett. 1992, 18, 427.

(35) Ogawa, T.; Sakamoto, N.; Hashimoto, T.; Han, C. D.; Baek, D. M. Macromolecules 1996, 29, 2113.

(36) Fried, H.; Binder, K. Europhys. Lett. 1991, 16, 237.

(37) Neutron Spectrrometers at Risø: http://www.risoe.dk/fys/ Equipment/Dr3/neuspec.htm.

(38) Neutronenstreuexperimenteam FRJ -2 in J ülich (the German and English text is available at Forschungzentrum J ülich, 1997).

(39) Pedersen, J . S.; Posselt, D.; Mortensen, K. J . Appl. Crystallogr. 1990, 23, 321.

(40) Almdal, K.; Mortensen, K.; Ryan, A. J .; Bates, F. S. Macromolecules 1996, 29, 5940.

(41) Balsara, N. P.; Fetters, L. J .; Hadjichristidis, N.; Lohse, D. J .; Han, C. C.; Graessley, W. W.; Krishnamoorti, R. Macromolecules 1992, 25, 6137.

MA0002545 